\title{
Mapping of QTLs Associated with Yield and Yield Related Traits in Durum Wheat (Triticum durum Desf.) Under Irrigated and Drought Conditions
}

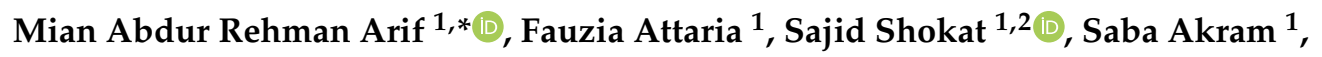 \\ Muhammad Qandeel Waheed ${ }^{1}$, Anjuman Arif ${ }^{1}$ and Andreas Börner ${ }^{3}$ \\ 1 Nuclear Institute for Agriculture and Biology, Jhang Road, Faisalabad 38000, Pakistan; \\ afsmta@gmail.com (F.A.); sajid_agrarian@yahoo.com (S.S.); sabaakram3463@yahoo.com (S.A.); \\ qandeel.nibgian@gmail.com (M.Q.W.); arifanjuman@gmail.com (A.A.) \\ 2 Department of Plant and Environmental Sciences, University of Copenhagen, Højbakkegård Allé 13, \\ DK-2630 Taastrup, Denmark \\ 3 Leibniz Institute of Plant Genetics and Crop Plant Research, Corrensstr. 3, Seeland OT, \\ 06466 Gatersleben, Germany; boerner@ipk-gatersleben.de \\ * Correspondence: m.a.rehman.arif@gmail.com
}

Received: 18 February 2020; Accepted: 11 March 2020; Published: 30 March 2020

\begin{abstract}
Global durum wheat consumption (Triticum durum Desf.) is ahead of its production. One reason for this is abiotic stress, e.g., drought. Breeding for resistance to drought is complicated by the lack of fast, reproducible screening techniques and the inability to routinely create defined and repeatable water stress conditions. Here, we report the first analysis of dissection of yield and yield-related traits in durum wheat in Pakistan, seeking to elucidate the genetic components of yield and agronomic traits. Analysis of several traits revealed a total of 221 (160 with logarithm of odds $(\mathrm{LOD})>2 \leq 3$ and 61 with LOD $>3$ ) quantitative trait loci (QTLs) distributed on all fourteen durum wheat chromosomes, of which 109 (78 with LOD $>2 \leq 3$ and 31 with LOD $>3$ ) were observed in 2016-17 (S1) and 112 (82 with LOD > $2 \leq 3$ and 30 with LOD > 3) were observed in 2017-18 (S2). Allelic profiles of yield QTLs on chromosome 2A and 7B indicate that allele A of Xgwm895 and allele B of Xbarc276 can enhance the Yd up to $6.16 \%$ in control and 5.27\% under drought. Moreover, if combined, a yield gain of up to $11 \%$ would be possible.
\end{abstract}

Keywords: Triticum durum; QTL mapping; drought stress; yield; stress indices; marker assisted selection

\section{Introduction}

Wheat has been the world's most important cereal from 10,000 years ago since the dawn of agriculture [1] up to the present day. With a production of $\sim 620$ million tons annually worldwide, wheat provides about $1 / 5$ th of the calories consumed by humans [2]. The current annual wheat yield increase is below 1\%, but it should be around $1.6 \%$ in order to meet the wheat demand (which is anticipated to rise by $60 \%$ with the world population, which will be 9 billion by 2050 [3].

Durum wheat (Triticum durum or Triticum turgidum subsp. durum), which is also referred to as pasta or macaroni wheat, is the hardest of all wheats. Its density, combined with its high protein content and gluten strength, make durum the wheat of choice for producing quality products including bread, couscous, frekeh, bulgur, and most importantly pasta [4]. A total of 9.3 and 10.5 million tons of pasta was produced in 2001 and 2003, respectively. By 2013, its production reached 13.5 million tons [5], providing an insight into the growing global demand for durum wheat. 
Durum wheat global consumption is ahead of its production. T. durum accounts for around $6 \%$ of total wheat production (37.7 MT in 2013; International Grain Council, October 2014) [6], occupying approximately 20 million hectares worldwide. High yielding cultivars endowed with drought tolerance and disease resistance, in addition to their high commercial and technological value, are therefore highly desirable. Durum wheat is primarily grown under rain-fed conditions where the frequent drought combined with heat stress is the major aspect of grain yield reduction [7-9]. Drought is one of the most severe stresses and is the main cause of significant losses in growth and productivity of crop plants [10]. About $45 \%$ of the 120 million hectares of land allocated in developing countries to wheat production is prone to drought [11]. Crop yield is seriously affected by water deficit in many growing areas of the world. It has been assessed that as much as $50 \%$ of the wheat cultivation area is regularly affected by drought [12].

In response to water deficit, morphological and biochemical changes are witnessed in plants which follow the functional damage and loss of important plant parts as the shortfall increases [13]. Various tolerance mechanisms (pigment content and stability and high relative water content) have been ascribed to the physiological changes in plants to water scarcity [14]. There are various ways in which drought-tolerant wheat species can be differentiated. These include growth response in water-limited conditions, alteration in water relations in tissues subjected to water stress, amassing of various ions, and early flowering [15]. As our comprehension of the physiological processes by which plants respond to water scarcity increases, we will be able to devise screening methods to select drought-tolerant accessions for further usage in breeding systems [16].

Generally, all yield components are disturbed under severe drought stress, most notably including fertile ears per unit area (reduction $\leq 60 \%$ ), seeds per spike (reduction $\leq 60 \%$ ), harvest index; and nitrogen harvest index (reduction $\leq 24 \%$ ), whereas lower grain weight is witnessed under mild stress [17]. Different cultivars differ in their responses to drought. Several factors $\left(\mathrm{CO}_{2}\right.$ exchange rate, stomatal conductance, relative water contents and osmotic changes) regulate the fate of drought resistance in a genotype and natural variation in these factors can be used as selection criteria to breed drought-tolerant cultivars [18]. Since the 1970s, the attained genetic gains in durum yield have been because of well-adjusted progress in fertility through distribution of relatively higher assimilates to the ear and the growing tillers which increased total biomass, whereas harvest index remained the same [19], probably because the ratio of improvement of grain yield to biological yield remained the same. Water usage of plants and minimization of the deleterious effects under water-scarce conditions can be modified by altering plant height, exposed leaf area and leaf index [20]. Moreover, varieties enriched with increased leaf turgidity and relative water contents in water-deficient environments have better yields in drought $[21,22]$.

Several factors are responsible for complications in breeding drought-tolerant cultivars, such as fast repeatable screening techniques and the incapacity to create drought conditions in a repeatable manner to screen large amounts of germplasm [23]. Breeders look for yield performance under drought stress. However, there are other factors that lead to poor yield in drought. To nullify this, drought indices have been devised based on plants performance under normal and drought conditions [24]. The drought resistance or susceptibility of a given genotype forms the basis of these indices [25]. Resistance to drought of a given genotype can be defined as the relative yield of that genotype in comparison to other genotypes that have been grown under the exact same stress conditions [26].

Different mapping populations have been made to investigate both biotic (disease resistance) and abiotic (drought, salinity) stresses [27,28], and many QTLs have been identified for yield and related traits in field trails. For example, two QTLs for test weight on chromosomes 7A and 6B (explaining $30 \%$ variation) and five QTLs of thousand kernel weight ( $\mathrm{Tkw}$ ) (explaining 32\% of total, including $25 \%$ genetic, variation) have been revealed [17]. Other investigations in durum wheat have reported QTLs for yield and its components and physiological developmental [29,30]. Although a plethora of information regarding genetic maps, populations and molecular markers exists, the transformation of this information into the breeding of drought-tolerant cultivars cannot yet be compared. This anomaly 
can be attributed to three reasons. The first is that yield, being a quantitative trait, has vague genetic architecture. The second is that the yield-linked loci are subject to a strong environmental influence. The third is the poor phenotyping of the concerned traits. Established QTLs in one environment often disappear in a different environment, and thus QTLs have been well-defined as constitutive versus adaptive. Nevertheless, robust QTLs of yield traits across 16 different environments on chromosomes $2 \mathrm{~B}$ and $3 \mathrm{~B}$ have been reported [29].

Here, we report the analysis of a bi-parental mapping population seeking to elucidate the genetic components of yield and agronomic traits under irrigated and drought conditions. Moreover, certain physiological traits were analyzed to check their response towards yield in both conditions. To add to this, various stress indices were calculated and their QTLs were sought. Thus, the current study was undertaken to achieve the following objectives: (1) to screen a panel of 114 recombinant inbred lines (RILs) of durum wheat for yield and yield-related traits under drought and irrigated conditions at the Nuclear Institute for Agriculture and Biology (NIAB), Pakistan; (2) to measure physiological traits and various stress indices of the population under irrigated and drought conditions; and (3) to uncover the potential QTLs of measured traits and their comparison.

\section{Results}

\subsection{Phenotypic Variation}

A three-way ANOVA revealed that treatment, i.e., drought, significantly reduced all the traits (Table 1). Moreover, germination (Gr), transpiration rate (Tr), stomatal conductance (Sc), photosynthetic rate (Phr), yield (Yd), harvest index (HI), and thousand kernel weight (Tkw) differed significantly at the genotype level. Impact of season was significant for Gr, Sc, Phr and Sl, where an increase for Gr was observed in S2, while Sc Phr and S1 were significantly higher in S1. Furthermore, genotype $x$ treatment interaction was significant for $\mathrm{Tr}, \mathrm{Sc}, \mathrm{Ph}$ and HI. Season $\mathrm{x}$ treatment had a significant effect on Gr, Sc, Phr, Wue, (heading time) Hd and Sl. Finally, genotype $x$ season $x$ treatment were significant for Sc and Yd.

Table 1. Range and mean \pm SD of traits measured in S1 and S2 including significant differences among genotypes, seasons, treatments, genotype $\mathrm{x}$ season, genotype $\mathrm{x}$ treatment, season $\mathrm{x}$ treatment and genotype $x$ season $x$ treatment interactions at $p$-values $0.05\left({ }^{*}\right), 0.01\left(^{* *}\right)$ and $0.001\left({ }^{* * *}\right)$ in durum wheat RIL population. $\mathrm{Gr}=$ germination $\%, \mathrm{Tr}=$ Transpiration rate, $\mathrm{Sc}=$ Stomatal conductance, $\mathrm{Phr}=$ Photosynthetic rate, Wue = water use efficiency, $\mathrm{Hd}=$ heading time, $\mathrm{Ph}=$ plant height, $\mathrm{Sl}=$ spike length, $\mathrm{Sw}=$ spike weight, $\mathrm{Sps}=$ seeds per spike, $\mathrm{Bm}=$ biomass, $\mathrm{Yd}=$ yield, $\mathrm{HI}=$ harvest index, $\mathrm{Tkw}=$ thousand kernel weight, $\mathrm{STI}=$ stress tolerance index, $\mathrm{MP}=$ mean productivity, $\mathrm{ST}=$ stress tolerance, SSI= stress susceptibility index, DRI = drought resistance index, S1 = season 1 (2016-17), S2 = (2017-18), $\mathrm{D}=$ drought, $\mathrm{R}=$ relative and $\mathrm{NA}=$ not applicable.

\begin{tabular}{|c|c|c|c|c|c|c|}
\hline \multicolumn{3}{|c|}{ S1 } & \multicolumn{3}{|c|}{ S2 } & \multirow{2}{*}{$p$-Value } \\
\hline Trait & Range & Mean \pm SD & Trait & Range & Mean \pm SD & \\
\hline \multicolumn{7}{|c|}{ Agronomic Traits } \\
\hline Gr_S1 & $50-100$ & $81.86 \pm 9.63$ & Gr_S2 & $80-100$ & $96.00 \pm 3.72$ & $\mathbf{P}_{\mathrm{S}}=* * *$ \\
\hline GrD_S1 & $47.5-100$ & $72.55 \pm 9.96$ & GrD_S2 & $70-100$ & $91.24 \pm 5.56$ & $\mathbf{P}_{\mathrm{T}}=* * *$ \\
\hline RGr_S1 & $66.7-100$ & $88.24 \pm 11.78$ & RGr_S2 & $73.68-100$ & $95.11 \pm 5.30$ & $\begin{array}{c}\mathbf{P}_{\mathrm{G}}={ }^{*} \\
\mathbf{P}_{\mathrm{S}^{*} \mathrm{~T}}=*\end{array}$ \\
\hline Hd_S1 & $80-128$ & $100.47 \pm 11.79$ & Hd_S2 & $84-120$ & $98.31 \pm 7.19$ & $\mathbf{P}_{\mathrm{T}}=* * *$ \\
\hline HdD_S1 & $80-126$ & $93.94 \pm 12.41$ & HdD_S2 & $78.5-114.5$ & $92.32 \pm 6.79$ & $\mathbf{P}_{\mathrm{S}^{*} \mathrm{~T}}={ }^{*}$ \\
\hline RHd_S1 & $51-121.78$ & $94.30 \pm 3.38$ & RHd_S2 & $80-101.66$ & $94.18 \pm 2.70$ & \\
\hline Ht_S1 & $61-148$ & $114.78 \pm 23.82$ & Ht_S2 & 62-185 & $111.36 \pm 26.31$ & $\mathbf{P}_{\mathbf{S}}=* * *$ \\
\hline HtD_S1 & $53-110$ & $80.24 \pm 12.45$ & HtD_S2 & $51-109.83$ & $83.65 \pm 14.14$ & $\mathbf{P}_{\mathrm{T}}=* * *$ \\
\hline RHt_S1 & $45.07-99.1$ & $74.14 \pm 10.05$ & RHt_S2 & $56.15-98.03$ & $76.51 \pm 8.94$ & \\
\hline
\end{tabular}


Table 1. Cont.

\begin{tabular}{|c|c|c|c|c|c|c|}
\hline \multicolumn{3}{|c|}{ S1 } & \multicolumn{3}{|c|}{$\mathrm{S} 2$} & \multirow{2}{*}{$p$-Value } \\
\hline Trait & Range & Mean \pm SD & Trait & Range & Mean \pm SD & \\
\hline \multicolumn{7}{|c|}{ Physiological Traits } \\
\hline Tr_S1 & $22.55-56.02$ & $37.38 \pm 6.86$ & Tr_S2 & $20.02-48.25$ & $34.17 \pm 6.51$ & $\mathbf{P}_{\mathrm{T}}=* * *$ \\
\hline TrD_S1 & $5.17-27.8$ & $15.56 \pm 4.65$ & TrD_S2 & 8.59-19.68 & $15.06 \pm 1.90$ & $\mathbf{P}_{\mathrm{G}}=* *$ \\
\hline RTr_S1 & $14.2-95.5$ & $42.98 \pm 15.98$ & RTr_S2 & $21.28-73.24$ & $45.43 \pm 9.78$ & $\mathbf{P}_{\mathrm{T}^{*} \mathrm{G}}=*$ \\
\hline Sc_S1 & $675.08-3743.6$ & $1492.70 \pm 527.26$ & Sc_S2 & $526.72-2059.5$ & $1143.90 \pm 293.57$ & $\mathbf{P}_{\mathrm{S}}=* * *$ \\
\hline ScD_S1 & $118.33-675.08$ & $342.96 \pm 114.03$ & ScD_S2 & $99.85-486.87$ & $262.53 \pm 93.35$ & $\mathbf{P}_{\mathrm{T}}=* * *$ \\
\hline RSc_S1 & $3.16-61.2$ & $25.29 \pm 11.39$ & RSc_S2 & $10.14-47.19$ & $23.50 \pm 8.17$ & $\begin{array}{c}\mathbf{P}_{\mathrm{G}}=* * * \\
\mathbf{P}_{\mathbf{S}^{*} \mathrm{~T}}=* * \\
\mathbf{P}_{\mathrm{T}^{* \mathrm{G}}}={ }^{* *} \\
\mathbf{P}_{\mathrm{S}^{*} \mathrm{~T}}={ }^{*}\end{array}$ \\
\hline Phr_S1 & $2.5-10.2$ & $3.97 \pm 1.24$ & Phr_S2 & $1.79-8.6$ & $3.49 \pm 1.25$ & $\mathbf{P}_{\mathrm{S}}=* * *$ \\
\hline PhrD_S1 & $1.25-3.84$ & $2.22 \pm 0.44$ & PhrD_S2 & $0.57-3.78$ & $1.72 \pm 0.67$ & $\mathbf{P}_{\mathrm{T}}=* * *$ \\
\hline RPhr_S1 & $14.93-93$ & $58.96 \pm 15.51$ & RPhr_S2 & $15.95-97.81$ & $50.94 \pm 15.76$ & $\begin{array}{c}\mathbf{P}_{\mathrm{G}}={ }^{*} \\
\mathbf{P}_{\mathrm{S}^{*} \mathrm{~T}}={ }^{* *}\end{array}$ \\
\hline Wue_S1 & $7.32-30.59$ & $11.89 \pm 4.03$ & Wue_S2 & $4.41-36.12$ & $11.14 \pm 6.30$ & $\mathbf{P}_{\mathbf{S}}=* *$ \\
\hline WueD_S1 & $5.95-54.63$ & $16.04 \pm 7.93$ & WueD_s2 & $3.29-31.33$ & $11.43 \pm 5.14$ & $\mathbf{P}_{\mathrm{T}}=* * *$ \\
\hline RWue_S1 & $30.56-659.29$ & $144.41 \pm 87.62$ & RWue_S2 & $24.38-225.63$ & $113.98 \pm 41.44$ & $\mathbf{P}_{\mathrm{S}^{*} \mathrm{~T}}=* *$ \\
\hline \multicolumn{7}{|c|}{ Spike Related Traits } \\
\hline S1_S1 & $6.4-12.06$ & $8.25 \pm 0.96$ & S1_S2 & $5.8-9.96$ & $7.60 \pm 0.71$ & $\mathbf{P}_{\mathrm{S}}=*$ \\
\hline SID_S1 & $4.43-9.58$ & $7.13 \pm 1.11$ & S1D_S2 & $4.4-9$ & $6.86 \pm 0.66$ & $\mathbf{P}_{\mathrm{T}}=* * *$ \\
\hline RS1_S1 & $56.51-99.43$ & $86.71 \pm 11.40$ & RS1_S2 & $74.48-100$ & $90.64 \pm 6.81$ & $\begin{array}{c}\mathbf{P}_{\mathbf{S}^{*} \mathrm{~T}}=* * * \\
\mathbf{P}_{\mathbf{T}^{*} \mathrm{G}}={ }^{*}\end{array}$ \\
\hline Sps_S1 & $24.6-62.6$ & $43.35 \pm 9.60$ & Sps_S2 & $32.8-91.3$ & $47.15 \pm 8.18$ & $\mathbf{P}_{\mathbf{T}}=* * *$ \\
\hline SpsD_S1 & $16.6-56.6$ & $33.44 \pm 7.75$ & SpsD_S2 & $15.8-48.1$ & $35.52 \pm 5.73$ & \\
\hline RSps_S1 & $43.24-99.44$ & $78.75 \pm 14.88$ & RSps_S2 & $34.35-99.75$ & $77.17 \pm 13.78$ & \\
\hline Sw_S1 & $1.45-4.94$ & $3.05 \pm 0.65$ & Sw_S2 & $1.58-4.96$ & $2.92 \pm 0.65$ & $\mathbf{P}_{\mathrm{T}}=* * *$ \\
\hline SwD_S1 & $1.19-3.76$ & $2.30 \pm 0.57$ & SwD_S2 & $1.28-3.66$ & $2.25 \pm 0.41$ & \\
\hline RSw_S1 & $42.41-100$ & $77.19 \pm 15.48$ & RSw_S2 & $43.2-99.66$ & $78.98 \pm 14.60$ & \\
\hline Tkw_S1 & $27.52-61.94$ & $48.88 \pm 5.30$ & Tkw_S2 & $31.09-56.4$ & $42.08 \pm 6.26$ & $\mathbf{P}_{\mathrm{T}}=* * *$ \\
\hline TkwD_S1 & $26.4-55.21$ & $45.07 \pm 4.95$ & TkwD_S2 & $26.53-54.87$ & $38.99 \pm 5.57$ & $\mathbf{P}_{\mathrm{G}}=* *$ \\
\hline RTkw_S1 & $67.96-99.91$ & $92.37 \pm 6.66$ & RTkw_S2 & $69.54-99.93$ & $92.20 \pm 5.66$ & \\
\hline \multicolumn{7}{|c|}{ Yield and Related Traits } \\
\hline Bm_S1 & $1180.8-6266.5$ & $3535.80 \pm 1086.20$ & Bm_S2 & $1120-9000$ & $3551.20 \pm 1172.60$ & $\mathbf{P}_{\mathrm{T}}=* * *$ \\
\hline BmD_S1 & $453.6-2282.6$ & $1070.50 \pm 349.64$ & BmD_S2 & $420-2320$ & $1191.40 \pm 373.78$ & $\mathbf{P}_{\mathrm{S}^{*} \mathrm{G}}={ }^{*}$ \\
\hline RBm_S1 & $10.03-92.01$ & $32.15 \pm 12.00$ & RBm_S2 & $12-62.42$ & $34.60 \pm 11.05$ & \\
\hline Yd_S1 & $305.68-1882.7$ & $1033.10 \pm 361.77$ & Yd_S2 & $306-1821$ & $1010.10 \pm 335.93$ & $\mathbf{P}_{\mathrm{T}}=* * *$ \\
\hline YdD_S1 & 120.73-639.19 & $350.92 \pm 109.75$ & YdD_S2 & $138-792$ & $332.46 \pm 116.73$ & $\mathbf{P}_{\mathrm{G}}=*$ \\
\hline RYd_S1 & $12.5-70.93$ & $36.39 \pm 12.21$ & RYd_S2 & $11.92-67.08$ & $34.89 \pm 12.27$ & $\begin{array}{l}\mathbf{P}_{\mathbf{S}^{*} \mathrm{G}}=* * * \\
\mathbf{P}_{\mathbf{S}^{*} \mathrm{~T}^{*} \mathrm{G}}=* *\end{array}$ \\
\hline HI_S1 & $11.58-66.6$ & $30.50 \pm 10.09$ & HI_S2 & $15.65-46.27$ & $28.56 \pm 6.38$ & $\mathbf{P}_{\mathrm{T}}=* * *$ \\
\hline HID_S1 & $14.16-60.49$ & $33.59 \pm 7.96$ & HID_S2 & $12.38-44$ & $28.47 \pm 6.68$ & $\mathbf{P}_{\mathrm{G}}=* * *$ \\
\hline RHI_S1 & $40.55-219.08$ & $117.66 \pm 36.37$ & RHI_S2 & $41.31-250.31$ & $103.30 \pm 30.84$ & $\mathbf{P}_{\mathrm{T}^{*} \mathrm{G}}=* * *$ \\
\hline \multicolumn{7}{|c|}{ Stress Indices } \\
\hline STI_S1 & $0.06-1.04$ & $0.36 \pm 0.20$ & STI_S2 & $0.06-1.41$ & $0.35 \pm 0.21$ & NA \\
\hline MP_S1 & $266.51-1236.5$ & $693.77 \pm 213.97$ & MP_S2 & 258-1306.5 & $674.98 \pm 198.37$ & NA \\
\hline ST_S1 & 158.19-1509 & $685.70 \pm 322.29$ & ST_S2 & 120-1401 & $684.24 \pm 297.06$ & NA \\
\hline SSI_S1 & $0.44-1.32$ & $0.96 \pm 0.18$ & SSI_S2 & $0.49-1.31$ & $0.97 \pm 0.18$ & NA \\
\hline DRI_S1 & $49.91-1255.7$ & $389.18 \pm 208.56$ & DRI_S2 & $49.97-1211.8$ & $371.11 \pm 229.50$ & NA \\
\hline
\end{tabular}




\subsection{Agronomic Traits}

Mean \pm SD along with range of the traits are provided in Table 1 , whereas box plots are given in Figure S1. Agronomic traits included Gr, Hd and Ph. S1 (2016-17) and S2 (2017-18) indicate different growing seasons. Mean germination under control in S1 reduced from $81.86 \pm 9.63$ (Gr_S1) to $72.55 \pm$ 9.96 (GrD_S1), with relative germination being $88.24 \pm 11.78$ (GrD_S1). Similarly, mean germination under control in S2 decreased from $96.00 \pm 3.72$ (Gr_S2) to $91.24 \pm 5.56$ (GrD_S2) in drought, with relative germination being $95.11 \pm 5.30$. Heading time in control in S1 decreased from $100.47 \pm 11.79$ (Hd_S1) to $93.94 \pm 12.419$ (HdD_S1) in drought. The same decreased from $100.47 \pm 11.79$ (Hd_S1) to $92.32 \pm 6.79$ (HdD_S2) under drought in S2. The relative heading times in S1 and S2 were $94.30 \pm 7.38$ and $94.18 \pm 2.70$, respectively. Reduction in plant height was also observed in drought as compared to control, as it was decreased from $114.78 \pm 23.82$ (Ph_S1) in control to $80.24 \pm 12.45$ (PhD_S1) in drought in S1. Likewise, it was decreased from $111.36 \pm 26.31$ (Ph_S2) to $83.65 \pm 14.14$ (PhD_S2) in drought in S2. (not mark in total)

\subsection{Physiological Traits}

These included $\mathrm{Tr}, \mathrm{Phr}, \mathrm{Sc}$ and Wue, which were higher in control than their corresponding values in drought in both seasons. In S1, Tr dropped form $37.38 \pm 6.86$ (Tr_S1) to $15.56 \pm 4.65$ (TrD_S1) under drought. On the same ground, in S2 it dropped from $34.17 \pm 6.51$ (Tr_S2) to $15.06 \pm 1.90$ (TrD_S2). However, RTr_S1 (42.98 \pm 15.98) was lower than RTr_S2 (45.43 \pm 9.78). Phr reduced from $3.97 \pm 1.24$ (Phr_S1) to $2.22 \pm 0.44$ (PhrD_S1) in drought. Likewise, a reduction from $3.49 \pm 1.25$ (Phr_S2) to $1.72 \pm$ 0.67 (PhD_S2) in drought was observed in S2. RPhr_S1 (58.96 \pm 15.51) was higher than RPhr_S2 (50.94 \pm 15.76). In control, Wue in control treatment in S1 was $11.89 \pm 4.03$ (Wue_S1), which increased to 16.04 \pm 7.93 in drought, whereas it was $11.14 \pm 6.30$ (Wue_S2) in control treatment in S2, which increased to $11.43 \pm 5.14$ (WueD_S2) in drought. This also raised the mean RWue_S1 $(144.41 \pm 87.62)$ and RWue_S2 $(113.98 \pm 41.44)$.

\subsection{Spike-Related Traits}

These included Sl, seeds per spike (Sps), spike weight (Sw) and Tkw. As per spike-related traits, the values of Sl, Sw and Tkw were higher in S1, whereas Sps was higher in S2. In drought, spike length was dropped to $7.13 \pm 1.11$ (SID_S1) from $8.25 \pm 0.96$ (S1_S1) in Sl, where RS1_S1 was 86.71 \pm 11.40 . On the other hand, in drought, $\mathrm{Sl}$ dropped to $6.86 \pm 0.66$ (SID_S2) from $7.60 \pm 0.71$ (S1_S2) with a relative value of $90.64 \pm 6.81$ in S2. Sps also witnessed a reduction in drought treatment which dropped from $43.35 \pm 9.60$ (Sps_S1) in S1 to $33.44 \pm 7.75$ (SpsD_S1) in drought. Sps_S2 was $47.15 \pm 8.18$ in control, which decreased to $35.52 \pm 5.73$ (SpsD_S2) in drought. RSps_S1 was (78.75 \pm 14.88 ), but was, however, higher than RSps_S2 (77.17 \pm 13.78). Mean Sw_S1 was 3.05 \pm 0.65 , whereas mean Sw_S2 was 2.92 \pm 0.65 . Both of them decreased to $2.30 \pm 0.57$ (SwD_S1) and 2.25 \pm 0.41 (SwD_S2) in drought. The corresponding relative values of Sw were $77.17 \pm 15.18$ (RSw_S1) and $78.98 \pm 14.60$ (RSw_S2), respectively. Mean Tkw_S1 (48.88 \pm 5.30) and mean Tkw_S2 (42.08 \pm 6.26) were reduced to $45.07 \pm$ 4.95 and $38.99 \pm 5.57$, respectively, in drought, whereas relative TKW values were comparable.

\subsection{Yield and Related Traits}

$\mathrm{Yd}$, biomass $(\mathrm{Bm})$ and $\mathrm{HI}$ were included in this section. The highest decrease among all traits from control to drought was observed for Bm, which decreased from $3535.80 \pm 1,086.20$ (Bm_S1) in S1 and $3551.20 \pm 1,172.60$ (Bm_S2) in S2 to $1070.50 \pm 349.64$ (BmD_S1) and $1191.40 \pm 373.78$ (BmD_S2), respectively. In line with the same trend, Yd in S1 dropped from 1,033.10 \pm 361.77 (Yd_S1) in control treatment to $350.92 \pm 109.75$ (YdD_S1) in drought. Likewise, Yd in S2 dropped from 1,010.10 \pm 335.93 (Yd_S2) in the control treatment to $332.46 \pm 116.73$ in the drought treatment in S2. An increasing trend in $\mathrm{HI}$ was observed from control to drought in S1. For example, HI in S1 in control was $30.50 \pm 10.09$, which increased to $33.59 \pm 7.96$ (HID_S1). In S2, HI in control and drought remained almost the same. 
Furthermore, there was very slight increase in relative HI during both seasons (RHI_S1 $=117.66 \pm$ 36.37, RHI_S2 $=103.30 \pm 30.84$ ).

\subsection{Stress Indices}

Altogether, the five stress indices, that is, stress tolerance index (STI), mean productivity (MP), stress tolerance (ST), stress susceptibility index (SSI), and drought resistance index (DRI), were close to each other in both S1 and S2. However, there were differences in their ranges. For example, STI_S1 ranged between 0.06 and 1.04, whereas STI_S2 ranged between 0.06 and 1.41. Likewise, MP_S1 ranged between 266.51 and 1236.5, whereas MP_S2 ranged between 258 and 1306.5. Ranges of ST_S1 and ST_S2 were 158.19-1509 and 120-1401, respectively. Similarly, ranges of SSI_S1 and SSI_S2 were 0.44-1.32 and 0.49-1.31, respectively. Finally, DRI_S1 ranged between 49.91 and 1225.7 and DRI_S2 ranged between 49.97 and 1211.8 .

There was a significant difference $(\alpha=0.05)$ observed between S1 and S2 with respect to trait values in control for $\mathrm{Gr}, \mathrm{Tr}, \mathrm{Sc}, \mathrm{Phr}, \mathrm{Sl}$, Sps and Tkw (data not shown). Moreover, there was also a significant difference $(\alpha=0.05)$ between S1 and S2 with respect to trait values under drought of $\mathrm{Gr}, \mathrm{Sc}$, $\mathrm{Phr}$, Wue, Ht, Sps, Tkw, Bm and HI. As far as relative values are concerned, significant differences $(\alpha=0.05)$ were observed between S1 and S2 for Gr, Phr, Wue, Ht, S1 and HI. However, there was no significant difference observed between the $\mathrm{S} 1$ and $\mathrm{S} 2$ trait values of all five drought indices (ST, MP, STI, SSI and DRI).

\subsection{Correlations}

As before, the data were divided into five sections for correlation analysis (relative values were excluded). These included (1) common agronomic traits ( $\mathrm{Gr}, \mathrm{Hd}$ and $\mathrm{Ph}$ ), (2) physiological traits (Tr, Sc, Phr and Wue), (3) spike-related traits (Sl, Sw, Sps, Tkw), (4) yield-related traits (Bm, Yd and HI) and (5) stress indices (STI, MP, ST, SSI and DRI). All sections were analyzed simultaneously for both S1 and S2. Data are shown in Tables S1-S5.

\subsection{QTL Mapping}

QTL mapping analysis of several traits revealed a total of 221 (160 with LOD $>2 \leq 3$ and 61 with LOD > 3) quantitative trait loci (QTLs) were distributed on all of the fourteen durum wheat chromosomes, of which 109 (78 with LOD $>2 \leq 3$ and 31 with LOD $>3$ ) were observed in S1 and 112 (82 with LOD $>2 \leq 3$ and 30 with LOD $>3$ ) were observed in S2. In S1, the donor of 54 QTLs was 'Omrabi 5' and the donor of other 55 QTLs was 'Belikh 2'. Likewise, in S2, the donor of 54 QTLs was 'Omrabi 5' and the donor of other 58 QTLs was 'Belikh 2'. These QTLs explained 5\%-33.73\% phenotypic variation in $\mathrm{S} 1$ and $5 \%-29.9 \%$ in S2. All details are given in Table 2.

From a chromosome perspective, the highest number of QTLs was observed on chromosome 5B (31 QTLs), followed by chromosomes 7B and 5A with 29 and 27 QTLs each, and by chromosomes 3B and $4 \mathrm{~B}$ with 21 and 20 QTLs, respectively. A total of 18 QTLs was detected on chromosome $3 \mathrm{~A}$, whereas 15 QTLs were detected on each of the chromosomes $1 \mathrm{~B}$ and 7A, and 14 QTLs resided on chromosome 2A. There were 10 and nine QTLs discovered on chromosomes 6B and 2B, respectively, whereas 6 QTLs detected on chromosome 6A and the least numbers of QTLs were detected on chromosomes $1 \mathrm{~A}$ and 4A (3 QTLs each).

\subsubsection{Agronomic Traits}

A total of 17 QTLs were detected for Gr on 9 of the 14 chromosomes (1B (2 QTLs), 3A, 3B, 4B, 5A (2 QTLs), 5B (6 QTLs), 6A, 6B (2 QTLs) and 7B) with five of them had an LOD > 3.0. There were two QTLs common, whereas the phenotypic variation explained ranged between $6 \%$ and $18 \%$. As far as $\mathrm{Hd}$ is concerned, there were 8 QTLs discovered on chromosomes 2A, 3A (2 QTLs), 5A (2 QTLs), 6B and 7B (2 QTLs), with three of them being highly significant. In addition, there was no QTL observed for Hd_S2 and RHd_S2. Altogether, QTLs explained 9\%-18\% of the variation in Hd. For Ph, a total of 19 
(4 highly significant) QTLs were revealed on chromosomes 2A, 2B (2 QTLs), 3A (2 QTLs), 4B (6 QTLs), $5 \mathrm{~A}$ (4 QTLs), 6A, 7A and 7B (2 QTLs) responsible for 6\%-22\% variation.

\subsubsection{Physiological Traits}

There were 14 QTLs identified for Tr across both seasons on chromosomes 2A, 2B, 3A (2 QTLs), 3B, 5A, 5B (3 QTLs), 6A and 7B (4 QTLs) where two QTLs (QTr_S2.NIAB-5B and QRTr_S2.NIAB-5B) were co-located. These QTLs explained 6-14\% variation. As regards to Sc, 9 QTLs were discovered on chromosomes 1B (2 QTLs), 2B, 3B, 5A, 5B, 6A (2 QTLs) and 7A responsible for 7-17\% variation and one QTL (QSc_S1.NIAB-1B.2) was highly significant. For Phr, 17 QTLs were found on all the chromosomes except $1 \mathrm{~A}$ and $4 \mathrm{~A}$. Among them, 7 QTLs were highly significant. The variation explained by these QTLs was 6-24\%. There were 11 QTLs uncovered on chromosomes 2A, 2B, 4B (3 QTLs), 5A (2 QTLs), $5 \mathrm{~B}, 7 \mathrm{~A}$ and $7 \mathrm{~B}$ (2 QTLs) for Wue from which three were highly significant, explaining $6 \%-22 \%$ of the phenotypic expression.

\subsubsection{Spike Related Traits}

Among spike related traits, there were 10 QTLs residing on chromosomes 2A, 2B, 3A, 4B (2 QTLs), 5B, 7A (2 QTLs) and 7B (2 QTLs) for Sl where 4 QTLs were highly significant. These QTLs explained $7-21 \%$ variation in Sl. Likewise, 18 QTLs were found for $\mathrm{Sw}$ that resided on chromosomes $1 \mathrm{~A}$ (2 QTLs), 2A (2 QTLs), 3A (3 QTLs), 3B (2 QTLs), 5A (3 QTLs), 5B (3 QTLs), 7A (2 QTLs) and 7B. With respect to Sps, 19 QTLs were discovered, which explained $6 \%-11 \%$ of the variation. These QTLs were lying on chromosomes 1A, 1B (3 QTLs), 3A (2 QTLs), 3B (3 QTLs), 4B, 5A, 5B (7 QTLs) and 7B. The 16 QTLs linked to Tkw were distributed on 8 different chromosomes (1B (3 QTLs), 2A, 4B (3 QTLs), 5A (3 QTLs), 5B, 6B (2 QTLs), 7A (2 QTLs) and 7B, of which only one was highly significant. All together, they explained $5 \%-12 \%$ of the variation.

\subsubsection{Yield and Related Traits}

A total of 19, 14, 16 and 12 QTLs were discovered for the yield related traits, respectively. The 19 QTLs of Bm were present on chromosomes 3A, 3B (3 QTLs), 4B, 5A, 5B (3 QTLs), 7A (3 QTLs) and 7B (7 QTLs). The 14 Yd QTLs were distributed on chromosomes 1B, 2A (3 QTLs), 3A, 3B (2 QTLs), 5A (2 QTLs), 5A, 5B, 6B (2 QTLs) and 7B (2 QTLs) explaining 6-30\% of phenotypic expression. There were three chromosomes, viz., 2A, 3B and 7B, where two Yd QTLs collocated. A total of seven chromosomes (1B, 2B, 3B (3 QTLs), 4B (2 QTLs), 5B (2 QTLs), 7A and 7B (2 QTLs) carried 12 HI QTLs which explained $7-11 \%$ phenotypic differences with only one highly significant QTL.

\subsubsection{Stress Indices}

A total of 18 QTLs were uncovered for various stress indices viz. STI (4 QTLs), MP (6 QTLs), ST (3 QTLs) and DRI (5 QTLs) on chromosomes 1B (2 QTLs), 2A, 2B, 3B (3 QTLs), 4A (3 QTLs), 5A $(5 \mathrm{QTLs}), 5 \mathrm{~B}, 6 \mathrm{~B}$ and $7 \mathrm{~A}$, which provided $8-26 \%$ of the variation among various stress indices.

Table 2. Composite interval mapping analysis of the durum wheat RIL mapping population. Highly significant LODs are in bold. QTL with similar superscripts are likely identical loci.

\begin{tabular}{|c|c|c|c|c|c|c|c|c|}
\hline Trait & QTL Designation & Chr & Pos & LOD & Closest Marker & Add. Effect & $\mathbf{R}^{2}$ & Donor \\
\hline \multicolumn{9}{|c|}{ Agronomic Traits } \\
\hline Gr_S2 & QGr_S2.NIAB-1B.1 & $1 \mathrm{~B}$ & 19.81 & 3.75 & Xgwm752 & -1.75 & 0.18 & B2 \\
\hline Gr_S2 & QGr_S2.NIAB-1B.2 & $1 \mathrm{~B}$ & 31.01 & 5.41 & Xbarc8 & -1.77 & 0.18 & B2 \\
\hline Gr_S2 & $\widetilde{Q} G r \_S 2 . N I A B-3 A^{S}$ & $3 \mathrm{~A}$ & 96.91 & 2.23 & Xbarc356 & 1.05 & 0.07 & O5 \\
\hline GrD_S2 & $\tilde{Q G r D \_S 2 . N I A B-3 B^{t}}$ & $3 \mathrm{~B}$ & 11.91 & 2.51 & Xgwm 285 & 1.59 & 0.07 & O5 \\
\hline GrD_S1 & $\widetilde{Q} G r D \_S 1 . N I A B-4 B^{a a}$ & $4 \mathrm{~B}$ & 36.51 & 2.04 & Xgwm710b & -2.64 & 0.06 & B2 \\
\hline Gr_S1 & QGr_S1.NIAB-5A.1 & $5 \mathrm{~A}$ & 73.71 & 2.51 & Xbarc342 & 3.06 & 0.08 & O5 \\
\hline Gr_S1 & $Q G r \_S 1 . N I A B-5 A .2^{h h}$ & $5 \mathrm{~A}$ & 171.81 & 3.59 & Xgwm1171b & -4.27 & 0.18 & B2 \\
\hline GrD_S1 & QGrD_S1.NIAB-5B & $5 \mathrm{~B}$ & 48.71 & 2.81 & Xgwm1165 & -3.31 & 0.1 & B2 \\
\hline
\end{tabular}


Table 2. Cont.

\begin{tabular}{|c|c|c|c|c|c|c|c|c|}
\hline Trait & QTL Designation & Chr & Pos & LOD & Closest Marker & Add. Effect & $\mathbf{R}^{2}$ & Donor \\
\hline GrD_S2 & QGrD_S2.NIAB-5B.1 ${ }^{n n}$ & $5 \mathrm{~B}$ & 207.51 & 2.6 & Xbarc266 & -1.71 & 0.08 & B2 \\
\hline RGr_S2 & QRGr_S2.NIAB-5B.1 $1^{n n}$ & $5 \mathrm{~B}$ & 207.51 & 2.87 & Xbarc266 & -2.02 & 0.1 & B2 \\
\hline GrD_S2 & QGrD_S2.NIAB-5B.2 & $5 \mathrm{~B}$ & 247.11 & 2.95 & Xbarc59 & -1.69 & 0.09 & B2 \\
\hline GrD_S2 & $\widetilde{Q G r D \_S 2 . N I A B-5 B .3^{p p}}$ & $5 \mathrm{~B}$ & 256.01 & 2.03 & Xgwm790 & -1.4 & 0.06 & B2 \\
\hline RGr_S2 & $\widetilde{Q} R G r \_S 2 . N I A B-5 B .2^{p p}$ & $5 \mathrm{~B}$ & 261.01 & 2.31 & Xgwm790 & -1.76 & 0.09 & B2 \\
\hline Gr_S2 & QGr_S2.NIAB-6B & $6 \mathrm{~A}$ & 121.51 & 3.09 & Xgwm4915 & 1.44 & 0.14 & O5 \\
\hline Gr_S1 & QGr_S1.NIAB-6B.1 ${ }^{r r}$ & $6 \mathrm{~B}$ & 141.41 & 2.9 & Xbarc178 & -3.64 & 0.12 & B2 \\
\hline Gr_S1 & $Q G r \_S 1 . N I A B-6 B .2$ & $6 \mathrm{~B}$ & 147.11 & 3.02 & Xbarc247 & -3.53 & 0.11 & B2 \\
\hline RGr_S1 & $\widetilde{Q R G} r \_S 1 . N I A B-7 B$ & $7 \mathrm{~B}$ & 216.21 & 2.25 & Xgwm983 & -3.69 & 0.08 & B2 \\
\hline HdD_S1 & QHdD_S1.NIAB-2A & $2 \mathrm{~A}$ & 5.81 & 2.21 & Xgwm1244 & -4.23 & 0.08 & B2 \\
\hline Hd_S1 & QHd_S1.NIAB-3Aq & $3 \mathrm{~A}$ & 73.51 & 4.54 & Xwmc50 & -5.11 & 0.17 & B2 \\
\hline HdD_S1 & QHdD_S1.NIAB-3A & $3 \mathrm{~A}$ & 44.51 & 2.1 & Xgwm779 & -4.47 & 0.09 & B2 \\
\hline Hd_S1 & $\widetilde{Q} H d \_S 1 . N I A B-5 A^{g g}$ & $5 \mathrm{~A}$ & 169.81 & 2.52 & Xbarc165 & -4.36 & 0.12 & B2 \\
\hline HdD_s2 & $\widetilde{Q H d D}-S 2 . N I A B-5 A^{h h}$ & $5 \mathrm{~A}$ & 188.01 & 3.39 & Xgwm1171b & -3.02 & 0.18 & B2 \\
\hline RHd_S1 & QRHĀ_S1.NIAB-6B & $6 \mathrm{~B}$ & 123.6 & 2.35 & Xgwm963a & 2.5 & 0.1 & O5 \\
\hline RHd_S1 & $\widetilde{Q R H d}$ dS1.NIAB-7B & $7 \mathrm{~B}$ & 42.5 & 4.35 & Xgwm195 & 4.02 & 0.16 & O5 \\
\hline RHd_S1 & QRHd_S1.NIAB-7B $B^{c c c}$ & $7 \mathrm{~B}$ & 223.8 & 2.05 & Xwmc396 & -2.3 & 0.08 & B2 \\
\hline PhD_S2 & $Q P h D \_S 2 . N I A B-2 A^{h}$ & $2 \mathrm{~A}$ & 121.31 & 2.04 & Xgwm71 & 3.64 & 0.06 & O5 \\
\hline Ph_S1 & $Q P h \_S 1 . N I A B-2 B^{l}$ & $2 \mathrm{~B}$ & 19.71 & 2.18 & Xwmc597 & 6.88 & 0.07 & O5 \\
\hline PhD_S1 & $\widetilde{Q P h D} \_S 1 . N I A B-3 A$ & $3 \mathrm{~A}$ & 14.61 & 2.86 & Xbarc294 & -3.99 & 0.09 & B2 \\
\hline PhD_S1 & $\widetilde{Q P h D \_S 1 . N I A B-2 B^{m}}$ & $2 \mathrm{~B}$ & 52.81 & 2.29 & Xgwm972 & -3.41 & 0.06 & B2 \\
\hline Ph_S2 & QRPh_S2.NIAB.3A & $3 \mathrm{~A}$ & 13.01 & 2.21 & Xbarc57 & 2.39 & 0.06 & B2 \\
\hline $\mathrm{Ph} \_\mathrm{S} 2$ & QRPh_S2.NIAB.4B & $4 \mathrm{~B}$ & 9.91 & 2.8 & Xbarc193 & -2.66 & 0.08 & B2 \\
\hline PhD_S2 & 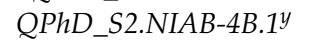 & $4 \mathrm{~B}$ & 14.91 & 2.92 & Xbarc193 & 4.78 & 0.1 & O5 \\
\hline PhD_S1 & QPhD_S1.NIAB-4B & $4 \mathrm{~B}$ & 15.91 & 5.2 & Xgwm925 & 6.15 & 0.22 & O5 \\
\hline Ph_s2 & $Q P h \_S 2 . N I A B-4 B .1^{z}$ & $4 \mathrm{~B}$ & 24.01 & 2.21 & Xgwm925 & 8.37 & 0.09 & O5 \\
\hline PhD_S2 & $Q P h D \_S 2 . N I A B-4 B .2^{z}$ & $4 \mathrm{~B}$ & 25.01 & 2.51 & Xgwm925 & 4.69 & 0.1 & O5 \\
\hline Ph_S2 & $\widetilde{Q P h} \_\bar{S} 2 . N I A B-4 B .2^{a a}$ & $4 \mathrm{~B}$ & 35.71 & 2.99 & Xgwm710b & 8.58 & 0.1 & O5 \\
\hline $\mathrm{Ph} \_\mathrm{S} 1$ & $\widetilde{Q P h} \_S 1 . N I A B-5 A^{e e}$ & $5 \mathrm{~A}$ & 46.91 & 2 & Xgwm129 & 6.04 & 0.06 & O5 \\
\hline PhD_S2 & QPhD_S2.NIAB-5A & $5 \mathrm{~A}$ & 277.71 & 2.3 & Xgwm126 & -4.36 & 0.09 & B2 \\
\hline Ph_S2 & $Q P h \_S 2 . N I A B-5 A^{j j}$ & $5 \mathrm{~A}$ & 334.41 & 2.42 & Xbarc261 & -7.91 & 0.08 & B2 \\
\hline RPh_S1 & $\widetilde{Q R P h} \_S 1 . N I A B-5 A$ & $5 \mathrm{~A}$ & 311.71 & 3.3 & Xgwm995 & 3.68 & 0.1 & O5 \\
\hline Ph_S1 & QPh_S1.NIAB-6A & $6 \mathrm{~A}$ & 51.31 & 3.16 & Xgwm4608 & -8.31 & 0.1 & B2 \\
\hline PhD_S2 & $\widetilde{Q P h D \_S 2 . N I A B-7 A}$ & $7 \mathrm{~A}$ & 144.21 & 2.04 & Xwmc488 & 3.68 & 0.06 & O5 \\
\hline RPh_S1 & $\widetilde{Q R P h} \_\bar{S} 1 . N I A B-7 B^{b b b}$ & $7 \mathrm{~B}$ & 252.61 & 2.92 & Xgwm112 & 3.84 & 0.09 & O5 \\
\hline $\mathrm{RPh} \_\mathrm{S} 2$ & $Q R P \bar{h} \_S 2 . N I A B .7 B$ & $7 \mathrm{~B}$ & 288.81 & 4.78 & Xwmc517 & 4.73 & 0.16 & O5 \\
\hline \multicolumn{9}{|c|}{ Physiological Traits } \\
\hline Tr_S1 & QTr_S1.NIAB-2A & $2 \mathrm{~A}$ & 189.71 & 2.8 & Xgwm382 & -2.41 & 0.11 & B2 \\
\hline TrD_S1 & $Q T r D \_S 1 . N I A B-2 B^{k}$ & $2 \mathrm{~B}$ & 0.01 & 4.13 & Xbarc183 & 1.9 & 0.12 & $\mathrm{O} 5$ \\
\hline RTr_S1 & QRTr_S1.NIAB-3A $q$ & $3 \mathrm{~A}$ & 73.51 & 3.49 & Xwmc50 & 5.87 & 0.13 & O5 \\
\hline TrD_S1 & QTrD_S1.NIAB-3A ${ }^{r}$ & $3 \mathrm{~A}$ & 77.51 & 3.48 & Xgwm5 & 1.74 & 0.13 & O5 \\
\hline RTr_S2 & QRTr_S2.NIAB.3B & $3 \mathrm{~B}$ & 152.31 & 2.2 & Xgwm655 & -11.61 & 0.14 & B2 \\
\hline Tr_S2 & QTr_S2.NIAB.5A $A^{i i}$ & $5 \mathrm{~A}$ & 274.71 & 2.41 & Xgwm126 & -1.1 & 0.07 & B2 \\
\hline TrD_S1 & $Q T r D \_S 1 . N I A B-5 B^{k k}$ & $5 \mathrm{~B}$ & 13.41 & 2.24 & Xgwm540a & -1.24 & 0.06 & B2 \\
\hline RTr_S2 & $\widetilde{Q R T r} \_S 2 . N I A B .5 B^{m m}$ & $5 \mathrm{~B}$ & 158.91 & 2.03 & Xgwm408 & -8.41 & 0.06 & B2 \\
\hline $\mathrm{Tr} \_\mathrm{S} 2$ & QTr_S2.NIAB.5B $B^{\mathrm{mm}}$ & $5 \mathrm{~B}$ & 158.11 & 2.9 & Xgwm408 & 1.34 & 0.09 & O5 \\
\hline Tr_S2 & QTr_S2.NIAB.6Aqq & $6 \mathrm{~A}$ & 246.21 & 2.55 & Xwmc621 & 1.45 & 0.12 & O5 \\
\hline TrD_S1 & QTrD_S1.NIAB-7B & $7 \mathrm{~B}$ & 51.11 & 3.07 & Xgwm400 & 1.68 & 0.11 & O5 \\
\hline TrD_S1 & QTrD_S1.NIAB-7B.2 $2^{a a a}$ & $7 \mathrm{~B}$ & 219.11 & 2.48 & Xbarc276 & -1.31 & 0.07 & B2 \\
\hline RTr_S1 & QRTr_S1.NIAB-7B $B^{c C C}$ & $7 \mathrm{~B}$ & 238.91 & 2.19 & Xwmc396 & 5.65 & 0.11 & O5 \\
\hline Tr_S1 & $Q T r \_S 1 . N I A B-7 B^{b b b}$ & $7 \mathrm{~B}$ & 248.91 & 3.03 & Xgwm112 & -2.69 & 0.14 & B2 \\
\hline Sc_S1 & QSc_S1.NIAB-1B.1 & $1 \mathrm{~B}$ & 168.61 & 2.47 & Xbarc61 & 168.28 & 0.08 & O5 \\
\hline Sc_S1 & QSc_S1.NIAB-1B.2 & $1 \mathrm{~B}$ & 192.41 & 5.03 & Xwmc134 & -245.34 & 0.17 & B2 \\
\hline ScD_S2 & $Q S c D \_S 2 . N I A B-2 B^{n}$ & $2 \mathrm{~B}$ & 107.81 & 2.17 & Xgwm47 & 1.81 & 0.07 & O5 \\
\hline ScD_S2 & QScD_S2.NIAB-3B ${ }^{u}$ & $3 \mathrm{~B}$ & 13.91 & 2.96 & Xgwm685 & -2.18 & 0.09 & B2 \\
\hline RSc_S2 & QRSc_S2.NIAB-5A & $5 \mathrm{~A}$ & 272.61 & 2.41 & Xgwm126 & 10.03 & 0.1 & O5 \\
\hline Sc_S2 & QSC_S2.NIAB-5B $B^{m m}$ & $5 \mathrm{~B}$ & 158.11 & 2.83 & Xgwm408 & 4.29 & 0.09 & O5 \\
\hline Sc_S2 & QSC_S2.NIAB-6A.1 & $6 \mathrm{~A}$ & 80.01 & 2.01 & Xbarc107 & -3.28 & 0.05 & B2 \\
\hline
\end{tabular}


Table 2. Cont.

\begin{tabular}{|c|c|c|c|c|c|c|c|c|}
\hline Trait & QTL Designation & Chr & Pos & LOD & Closest Marker & Add. Effect & $\mathbf{R}^{2}$ & Donor \\
\hline Sc_S2 & $Q S c \_S 2 . N I A B-6 A .2^{q q}$ & $6 \mathrm{~A}$ & 244.21 & 2.44 & Xwmc621 & 4.88 & 0.13 & O5 \\
\hline ScD_S1 & $\widetilde{Q S c D} \_S 1 . N I A B-7 A^{t t}$ & $7 \mathrm{~A}$ & 0.01 & 2.92 & Xgwm1171a & 37.93 & 0.09 & O5 \\
\hline RPhr_S2 & QRPhr_S2.NIAB-1B $B^{d}$ & $1 \mathrm{~B}$ & 140.81 & 3.65 & Xwmc156 & 6.26 & 0.15 & O5 \\
\hline PhrD_S2 & QPhrD_S2.NIAB-2A.1 ${ }^{h}$ & $2 \mathrm{~A}$ & 121.31 & 3.15 & Xgwm71 & 0.14 & 0.1 & O5 \\
\hline PhrD_S2 & $\widetilde{Q} P h r D \_S 2 . N I A B-2 A .2^{j}$ & $2 \mathrm{~A}$ & 175.21 & 5.41 & Xgwm1256 & -0.25 & 0.24 & $\mathrm{~B} 2$ \\
\hline RPhr_S2 & QRPhr_S2.NIAB-2Bl & $2 \mathrm{~B}$ & 18.71 & 2.25 & Xwmc597 & 4.63 & 0.07 & O5 \\
\hline RPhr_S2 & $\widetilde{Q R P h r} \_\bar{S} 2 . N I A B-3 A .1^{\circ}$ & $3 \mathrm{~A}$ & 17.61 & 3.68 & Xgwm757 & -5.44 & 0.12 & $\mathrm{~B} 2$ \\
\hline Phr_S2 & QPhr_S2.NIAB-3A $A^{r}$ & $3 \mathrm{~A}$ & 77.51 & 4.5 & Xgwm5 & 0.12 & 0.17 & O5 \\
\hline RPhr_S2 & QRPhr_S2.NIAB-3A.2 & $3 \mathrm{~A}$ & 108.01 & 2.36 & Xgwm666 & -4.58 & 0.07 & $\mathrm{~B} 2$ \\
\hline PhrD_S2 & QPhrD_S2.NIAB-3B.1 ${ }^{w}$ & $3 \mathrm{~B}$ & 181.81 & 3.75 & Xgwm299 & -0.15 & 0.13 & B2 \\
\hline PhrD_S2 & QPhrD_S2.NIAB-3B.2 ${ }^{w}$ & 3B & 186.81 & 2.82 & Xgwm299 & -0.14 & 0.1 & B2 \\
\hline Phr_S1 & $Q P h r \_S 1 . N I A B-4 B^{b b}$ & $4 \mathrm{~B}$ & 43.31 & 2.34 & Xgwm1167 & -0.34 & 0.07 & B2 \\
\hline Phr_S2 & QPhr_S2.NIAB-5B & $5 B$ & 6.41 & 4.5 & Xgwm191 & 0.09 & 0.14 & O5 \\
\hline Phr_S1 & QPhr_S1.NIAB-6A & $6 \mathrm{~A}$ & 2.01 & 2.15 & Xgwm459 & -0.31 & 0.06 & $\mathrm{~B} 2$ \\
\hline Phr_S1 & QPhr_S1.NIAB-6B & $6 \mathrm{~B}$ & 0.01 & 2.47 & Xgwm940b & -0.37 & 0.08 & $\mathrm{~B} 2$ \\
\hline RPhr_S2 & QRPhr_S2.NIAB-6B & $6 \mathrm{~B}$ & 60.81 & 2.09 & Xbarc136 & 8.31 & 0.28 & O5 \\
\hline Phr_S2 & QPhr_S2.NIAB-7A & $7 \mathrm{~A}$ & 186.41 & 3.14 & $X g w m 276$ & -0.09 & 0.13 & B2 \\
\hline PhrD_S2 & $\widetilde{Q P h r D} \_S 2 . N I A B-7 B$ & $7 \mathrm{~B}$ & 106.81 & 2.06 & Xwmc182 & 0.12 & 0.06 & O5 \\
\hline RPhr_S2 & $\widetilde{Q R P h r}{ }_{-S} 2 . N I A B-7 B^{x x}$ & $7 \mathrm{~B}$ & 150.61 & 2.31 & Xgwm46 & -4.71 & 0.08 & B2 \\
\hline WueD_s2 & QWueD_S2.NIAB-2 $A^{j}$ & $2 \mathrm{~A}$ & 166.21 & 2.33 & Xgwm1256 & -1.56 & 0.19 & B2 \\
\hline WueD_S1 & QWueD_S1.NIAB-2B ${ }^{k}$ & $2 \mathrm{~B}$ & 0.01 & 3.71 & Xbarc183 & -0.03 & 0.11 & B2 \\
\hline WueD_S1 & QWueD_S1.NIAB-4B & $4 \mathrm{~B}$ & 31.71 & 2.6 & Xbarc199 & -0.02 & 0.08 & B2 \\
\hline Wue_S1 & QWue_S1.NIAB-4B.1 ${ }^{a a}$ & $4 \mathrm{~B}$ & 36.51 & 3.06 & $X g w m 710 b$ & -0.01 & 0.1 & B2 \\
\hline Wue_S1 & QWue_S1.NIAB-4B.2bb & $4 \mathrm{~B}$ & 43.31 & 2.12 & Xgwm1167 & -0.01 & 0.07 & B2 \\
\hline WueD_S2 & QWueD_S2.NIAB-5A & $5 \mathrm{~A}$ & 259.01 & 4.5 & Xwmc727 & 1.88 & 0.22 & O5 \\
\hline Wue_S2 & QWue_S2.NIAB-5A & $5 \mathrm{~A}$ & 269.61 & 2 & Xgwm126 & 0.93 & 0.08 & O5 \\
\hline Wue_S2 & $\tilde{Q W u e \_S 2 . N I A B-5 B^{m m}}$ & $5 B$ & 158.91 & 2.12 & Xgwm408 & -0.88 & 0.06 & B2 \\
\hline Wue_S1 & QWue_S1.NIAB-7A & $7 \mathrm{~A}$ & 18.01 & 2.69 & Xwmc283 & -0.01 & 0.09 & B2 \\
\hline WueD_S2 & QWueD_S2.NIAB-7B ${ }^{x x}$ & $7 \mathrm{~B}$ & 149.61 & 2 & Xgwm46 & -0.86 & 0.06 & B2 \\
\hline Wue_S1 & QWue_S1.NIAB-7B & $7 \mathrm{~B}$ & 329.61 & 2.08 & Xbarc32 & 0.01 & 0.07 & O5 \\
\hline \multicolumn{9}{|c|}{ Spike Related Traits } \\
\hline S1_S1 & $Q S I \_S 1 . N I A B-2 A^{g}$ & $2 \mathrm{~A}$ & 76.41 & 6.89 & Xgwm122 & 0.46 & 0.21 & O5 \\
\hline SlD_S1 & QSID_S1.NIAB-2B & $2 \mathrm{~B}$ & 33.21 & 4.23 & Xbarc55 & -0.57 & 0.16 & $\mathrm{~B} 2$ \\
\hline RS1_S1 & QRSI_S1.NIAB-3A 9 & $3 \mathrm{~A}$ & 61.11 & 2.1 & Xwmc50 & -5.64 & 0.13 & B2 \\
\hline SID_S2 & QSID_S2.NIAB-4B $B^{c c}$ & $4 \mathrm{~B}$ & 151.81 & 2.34 & Xgwm940a & -0.18 & 0.08 & B2 \\
\hline Sl_S1 & QSI_S1.NIAB-4B & $4 \mathrm{~B}$ & 154.81 & 3.8 & Xgwm935a & -0.33 & 0.19 & B2 \\
\hline S1_S2 & QSI_S2.NIAB-5B & $5 \mathrm{~B}$ & 33.31 & 3.04 & Xgwm1180 & -0.24 & 0.1 & B2 \\
\hline S1D_S2 & QSID_S2.NIAB-7A & $7 \mathrm{~A}$ & 217.81 & 2.13 & Xgwm332 & -0.17 & 0.07 & $\mathrm{~B} 2$ \\
\hline S1_S2 & QSI_S2.NIAB-7A & $7 \mathrm{~A}$ & 278.21 & 4.05 & Xgwm1061 & -0.27 & 0.14 & $\mathrm{~B} 2$ \\
\hline RS1_S2 & $Q R S I \_S 2 . N I A B-7 B^{w w}$ & $7 \mathrm{~B}$ & 89.91 & 3.86 & Xgwm573 & 3.11 & 0.18 & O5 \\
\hline RS1_S1 & QRSI_S1.NIAB-7B & $7 \mathrm{~B}$ & 117.61 & 2.38 & Xgwm1184 & -5.05 & 0.1 & $\mathrm{~B} 2$ \\
\hline SwD_S1 & QSwD_S1.NIAB-1 $A^{a}$ & $1 \mathrm{~A}$ & 27.11 & 2.71 & Xwmc312 & 0.18 & 0.09 & O5 \\
\hline Sw_S1 & QSw_S1.NIAB-1A & $1 \mathrm{~A}$ & 30.11 & 5.25 & Xwmc93a & 0.29 & 0.19 & O5 \\
\hline SwD_S1 & QS $w D \_S 1 . N I A B-2 A^{g}$ & $2 \mathrm{~A}$ & 76.41 & 2.22 & Xgwm122 & 0.14 & 0.06 & O5 \\
\hline RSw_S1 & $\widetilde{Q} R S w \_S 1 . N I A B-2 A^{g}$ & $2 \mathrm{~A}$ & 76.41 & 3.38 & Xgwm122 & 5.1 & 0.1 & O5 \\
\hline SwD_S1 & 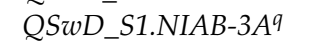 & $3 \mathrm{~A}$ & 68.51 & 2.55 & Xwmc50 & -0.16 & 0.08 & $\mathrm{~B} 2$ \\
\hline RSw_S1 & $Q R S w \_S 1 . N I A B-3 A^{q}$ & $3 \mathrm{~A}$ & 72.51 & 3.02 & Xwmc50 & -5.64 & 0.12 & $\mathrm{~B} 2$ \\
\hline Sw_S1 & $Q S w \_S 1 . N I A B-3 A^{s}$ & $3 \mathrm{~A}$ & 97.81 & 2.62 & Xbarc356 & 0.2 & 0.08 & O5 \\
\hline SwD_S1 & $Q S w D \_S 1 . N I A B-3 B^{u}$ & 3B & 13.91 & 2.82 & Xgwm685 & 0.18 & 0.08 & O5 \\
\hline RSw_S1 & $Q R S w \_S 1 . N I A B-3 B^{w}$ & 3B & 194.81 & 2.28 & Xgwm299 & -5.65 & 0.12 & B2 \\
\hline SwD_S1 & $Q S w D \_S 1 . N I A B-5 A^{d d}$ & $5 \mathrm{~A}$ & 0.01 & 2.94 & Xgwm154 & 0.18 & 0.09 & O5 \\
\hline SwD_S2 & $Q S w D \_S 2 . N I A B-5 A^{i i}$ & $5 \mathrm{~A}$ & 277.71 & 2.14 & Xgwm126 & -0.12 & 0.09 & B2 \\
\hline RSw_S1 & $Q R S w \_S 1 . N I A B-5 A^{j j}$ & $5 \mathrm{~A}$ & 337.41 & 3.1 & Xbarc261 & -5.74 & 0.13 & $\mathrm{~B} 2$ \\
\hline RSw_S2 & $Q R S w \_S 2 . N I A B-5 B .1^{\circ o}$ & $5 B$ & 237.11 & 2.42 & Xgwm1072 & 4.68 & 0.09 & O5 \\
\hline RSw_S2 & $\widetilde{Q} R S w \_S 2 . N I A B-5 B .2^{p p}$ & $5 B$ & 260.01 & 3.65 & Xgwm790 & 5.87 & 0.15 & O5 \\
\hline SwD_S1 & $Q S w D \_S 1 . N I A B-5 B$ & $5 B$ & 270.91 & 2.23 & Xgwm605 & 0.14 & 0.06 & O5 \\
\hline SwD_S2 & $Q S w D \_S 2 . N I A B-7 A^{u u}$ & $7 \mathrm{~A}$ & 57.81 & 2 & Xwmc405 & -0.12 & 0.08 & $\mathrm{~B} 2$ \\
\hline RSw_S1 & $Q R S w \_S 1 . N I A B-7 A$ & $7 \mathrm{~A}$ & 98.81 & 2.59 & $X g w m 710 a$ & -4.37 & 0.07 & $\mathrm{~B} 2$ \\
\hline
\end{tabular}


Table 2. Cont.

\begin{tabular}{|c|c|c|c|c|c|c|c|c|}
\hline Trait & QTL Designation & Chr & Pos & LOD & Closest Marker & Add. Effect & $\mathbf{R}^{2}$ & Donor \\
\hline RSw_S1 & $Q R S w \_S 1 . N I A B-7 B^{w w}$ & $7 \mathrm{~B}$ & 92.21 & 3.09 & Xgwm573 & 5.36 & 0.09 & O5 \\
\hline SpsD_S1 & QSpsD_S1.NIAB-1 $A^{a}$ & $1 \mathrm{~A}$ & 25.11 & 2.41 & Xwmc312 & 2.11 & 0.06 & O5 \\
\hline RSps_S1 & QRSps_S1.NIAB-1BC & 1B & 104.61 & 2.12 & Xwmc419 & -4.03 & 0.06 & B2 \\
\hline Sps_S1 & QSps_S1.NIAB-1B. $1^{d}$ & $1 \mathrm{~B}$ & 143.81 & 2.5 & Xwmc156 & 3.22 & 0.11 & O5 \\
\hline Sps_S1 & QSps_S1.NIAB-1B.2 & 1B & 154.61 & 2.75 & Xwmc548 & 2.91 & 0.08 & O5 \\
\hline Sps_S2 & QSps_S2.NIAB-3A $A^{p}$ & $3 \mathrm{~A}$ & 56.11 & 2.5 & Xbarc45 & 2.63 & 0.1 & O5 \\
\hline SpsD_S1 & QSpsD_S1.NIAB-3A $A^{q}$ & $3 \mathrm{~A}$ & 68.51 & 3.6 & Xwmc50 & -2.6 & 0.1 & B2 \\
\hline Sps_S1 & QSps_S1.NIAB-3B & $3 \mathrm{~B}$ & 8.31 & 2 & Xgwm625 & 2.4 & 0.06 & O5 \\
\hline SpsD_S1 & QSpsD_S1.NIAB-3B ${ }^{u}$ & $3 B$ & 13.91 & 3.61 & Xgwm685 & 2.67 & 0.1 & O5 \\
\hline RSps_S1 & QRSps_S1.NIAB-3B ${ }^{w}$ & 3B & 182.81 & 2.63 & Xgwm299 & 4.51 & 0.08 & O5 \\
\hline Sps_S2 & QSps_S2.NIAB-4B & $4 \mathrm{~B}$ & 155.91 & 2.94 & Xwmc428 & -3.29 & 0.09 & B2 \\
\hline SpsD_S1 & QSpsD_S1.NIAB-5A $A^{d d}$ & $5 \mathrm{~A}$ & 1.01 & 2.84 & Xgwm154 & 2.43 & 0.08 & O5 \\
\hline Sps_S1 & QSps_S1.NIAB-5B.1 $1^{k k}$ & $5 \mathrm{~B}$ & 13.41 & 2.3 & Xgwm540a & -3.34 & 0.07 & B2 \\
\hline RSps_S1 & QRSps_S1.NIAB-5B ${ }^{l l}$ & $5 B$ & 132.21 & 4.41 & Xgwm1043 & 8.72 & 0.33 & O5 \\
\hline RSps_S2 & QRSps_S2.NIAB-5B.2 $2^{\text {ll }}$ & $5 B$ & 143.21 & 2.14 & Xgwm1043 & 3.9 & 0.07 & O5 \\
\hline SpsD_S2 & QSpsD_S2.NIAB-5B & $5 B$ & 218.31 & 3.22 & Xbarc232 & 2 & 0.11 & O5 \\
\hline RSps_S2 & QRSps_S2.NIAB-5B.20o & $5 B$ & 232.71 & 2.83 & Xgwm1072 & 4.41 & 0.09 & O5 \\
\hline Sps_S2 & QSps_S2.NIAB-5B & $5 B$ & 233.11 & 2.27 & Xgwm1072 & -2.17 & 0.07 & B2 \\
\hline Sps_S1 & QSps_S1.NIAB-5B.2 & $5 B$ & 273.91 & 2.85 & Xbarc243 & 2.9 & 0.09 & O5 \\
\hline SpsD_S2 & QSpsD_S2.NIAB-7B & $7 \mathrm{~B}$ & 172.61 & 2.1 & Xwmc218 & -2.36 & 0.07 & B2 \\
\hline RTkw_S1 & $Q R T k w \_S 1 . N I A B-1 B^{c}$ & $1 \mathrm{~B}$ & 102.61 & 2.05 & Xwmc419 & -2.01 & 0.08 & B2 \\
\hline TkwD_S1 & QTkwD_S1.NIAB-1B.1 & $1 \mathrm{~B}$ & 221.11 & 2.26 & Xgwm 268 & 1.56 & 0.09 & O5 \\
\hline TkwD_S1 & QTkwD_S1.NIAB-1B.2 & $1 \mathrm{~B}$ & 230.31 & 2.21 & Xgwm153 & 1.32 & 0.06 & O5 \\
\hline Tkw_S1 & QTkw_S1.NIAB-2Af & $2 \mathrm{~A}$ & 17.51 & 2.11 & Xgwm726 & 1.88 & 0.08 & O5 \\
\hline RTkw_S2 & QRTkw_S2.NIAB-4B.1 $1^{a a}$ & $4 \mathrm{~B}$ & 35.71 & 3.98 & $X g w m 710 b$ & -1.93 & 0.11 & $\mathrm{~B} 2$ \\
\hline Tkw_S2 & $Q T k w \_S 2 . N I A B-4 B^{a a}$ & $4 \mathrm{~B}$ & 35.71 & 2.74 & Xgwm710b & 1.98 & 0.09 & O5 \\
\hline RTkw_S2 & QRTkw_S2.NIAB-4B.2 $2^{b b}$ & $4 \mathrm{~B}$ & 42.51 & 4.24 & Xgwm1167 & -1.98 & 0.12 & B2 \\
\hline RTkw_S1 & QRTkw_S1.NIAB-5Aff & $5 \mathrm{~A}$ & 124.71 & 3.22 & Xgwm1236 & -2.51 & 0.12 & B2 \\
\hline TkwD_S1 & QTkwD_S1.NIAB-5Aff & $5 \mathrm{~A}$ & 126.71 & 2 & Xgwm1236 & -1.38 & 0.07 & $\mathrm{~B} 2$ \\
\hline TkwD_S2 & QTkwD_S2.NIAB-5A & $5 \mathrm{~A}$ & 277.71 & 2.62 & Xgwm126 & -1.85 & 0.1 & $\mathrm{~B} 2$ \\
\hline RTkw_S2 & QRTkw_S2.NIAB-5B & $5 B$ & 62.01 & 2.01 & Xbarc128 & -1.38 & 0.05 & B2 \\
\hline Tkw_S1 & $\widetilde{Q} T k w \_\bar{S} 1 . N I A B-6 B^{r r}$ & $6 \mathrm{~B}$ & 144.11 & 2.55 & Xbarc178 & 1.86 & 0.08 & O5 \\
\hline TkwD_S1 & QTkwD_S1.NIAB-6Bs & $6 \mathrm{~B}$ & 160.61 & 2.74 & Xgwm889 & 1.61 & 0.09 & O5 \\
\hline RTkw_S2 & QRTkw_S2.NIAB-7A & $7 \mathrm{~A}$ & 113.41 & 2.14 & Xwmc603 & -1.35 & 0.05 & B2 \\
\hline Tkw_S1 & QTkw_S1.NIAB-7A vv & $7 \mathrm{~A}$ & 220.81 & 2.93 & Xgwm332 & 2.03 & 0.1 & O5 \\
\hline RTkw_S1 & QRTkw_S1.NIAB-7B & $7 \mathrm{~B}$ & 275.21 & 2.66 & Xbarc258 & -2.09 & 0.09 & $\mathrm{~B} 2$ \\
\hline \multicolumn{9}{|c|}{ Yield and Related Traits } \\
\hline RBm_S1 & QRBm_S1.NIAB-3 $A^{o}$ & $3 \mathrm{~A}$ & 16.61 & 2.32 & Xgwm757 & -1.29 & 0.06 & B2 \\
\hline RBm_S1 & QRBm_S1.NIAB-3B ${ }^{t}$ & $3 \mathrm{~B}$ & 11.91 & 2.06 & Xgwm285 & -1.22 & 0.05 & B2 \\
\hline RBm_S2 & $\widetilde{Q R B m} \_S 2 . N I A B-3 B^{v}$ & 3B & 88.11 & 2.85 & Xbarc344 & 3.88 & 0.1 & O5 \\
\hline Bm_s2 & QBm_S2.NIAB-3B & 3B & 89.11 & 2.22 & Xbarc344 & -349.5 & 0.07 & B2 \\
\hline BmD_S1 & $Q B m D \_S 1 . N I A B-4 B$ & $4 \mathrm{~B}$ & 88.31 & 3.56 & Xgwm998 & 130.01 & 0.11 & O5 \\
\hline RBm_S1 & QRBm_S1.NIAB-5A & $5 \mathrm{~A}$ & 46.91 & 2.4 & Xgwm129 & -1.39 & 0.07 & B2 \\
\hline Bm_S2 & QBm_S2.NIAB-5B & $5 B$ & 232.71 & 2.65 & Xgwm1072 & -389.16 & 0.08 & B2 \\
\hline RBm_S2 & QRBm_S2.NIAB-5B ${ }^{\circ o}$ & $5 B$ & 232.71 & 2.03 & Xgwm1072 & 3.04 & 0.07 & O5 \\
\hline RBm_S1 & QRBm_S1.NIAB-5Bpp & $5 B$ & 259.01 & 3.45 & Xgwm790 & -1.78 & 0.11 & B2 \\
\hline RBm_S1 & QRBm_S1.NIAB-7A & $7 \mathrm{~A}$ & 213.91 & 2.83 & Xbarc253 & -1.46 & 0.08 & B2 \\
\hline RBm_S2 & $\widetilde{Q} R B m \_S 2 . N I A B-7 A$ & $7 \mathrm{~A}$ & 91.51 & 2.21 & Xbarc1025 & -3.38 & 0.08 & B2 \\
\hline BmD_S2 & $\widetilde{Q B m D \_S} 2 . N I A B-7 A^{u u}$ & $7 \mathrm{~A}$ & 52.81 & 2.73 & Xwmc405 & -150.39 & 0.13 & B2 \\
\hline Bm_S1 & QBm_S1.NIAB-7B.1 & $7 \mathrm{~B}$ & 137.51 & 3.06 & $X g w m 540 b$ & -558.27 & 0.1 & B2 \\
\hline Bm_S1 & QBm_S1.NIAB-7B.2 & $7 \mathrm{~B}$ & 164.41 & 3.16 & Xwmc476 & 640.83 & 0.13 & O5 \\
\hline RBm_S2 & QRBm_S2.NIAB-7B.1yy & $7 \mathrm{~B}$ & 172.61 & 3.55 & Xwmc218 & -4.24 & 0.12 & B2 \\
\hline RBm_S2 & QRBm_S2.NIAB-7B.2 & 7B & 179.41 & 3.39 & Xgwm963b & -4.14 & 0.11 & B2 \\
\hline RBm_S2 & QRBm_S2.NIAB-7B.3 $3^{z z}$ & $7 \mathrm{~B}$ & 184.41 & 2.43 & Xgwm1085 & -3.75 & 0.08 & B2 \\
\hline Bm_S1 & $Q B m \_S 1 . N I A B-7 B .3^{a a a}$ & $7 \mathrm{~B}$ & 218.21 & 2 & Xbarc276 & -334.54 & 0.07 & B2 \\
\hline BmD_S2 & $\widetilde{Q B m D} \_S 2 . N I A B-7 B^{a a a}$ & $7 \mathrm{~B}$ & 221.11 & 2 & Xbarc276 & -113.28 & 0.07 & B2 \\
\hline RYd_S2 & $Q R Y d \_S 2 . N I A B-1 B^{b}$ & $1 \mathrm{~B}$ & 95.61 & 2.07 & Xgwm784 & 3.61 & 0.06 & O5 \\
\hline YdD_S1 & QYdD_S1.NIAB-2Af & $2 \mathrm{~A}$ & 26.01 & 2.65 & Xgwm 726 & 37.77 & 0.09 & O5 \\
\hline YdD_S2 & $\widetilde{Q} Y d D \_S 2 . N I A B-2 A^{i}$ & $2 \mathrm{~A}$ & 124.61 & 2.51 & Xgwm895 & 34.89 & 0.08 & O5 \\
\hline
\end{tabular}


Table 2. Cont.

\begin{tabular}{|c|c|c|c|c|c|c|c|c|}
\hline Trait & QTL Designation & Chr & Pos & LOD & Closest Marker & Add. Effect & $\mathbf{R}^{2}$ & Donor \\
\hline RYd_S1 & QRYd_S1.NIAB-2A $A^{i}$ & $2 \mathrm{~A}$ & 125.21 & 2.44 & Xgzm895 & -4.07 & 0.07 & B2 \\
\hline Yd_S2 & $\widetilde{Q} Y d \_S 2 . N I A B-3 A^{p}$ & $3 \mathrm{~A}$ & 52.11 & 2.38 & Xbarc 45 & 96.11 & 0.07 & O5 \\
\hline RYd_s2 & QRYd__S2.NIAB-3B ${ }^{x}$ & 3B & 205.71 & 3.58 & Xgwm547 & -4.91 & 0.11 & B2 \\
\hline Yd_S2 & $Q Y d \_S 2 . N I A B-3 B^{x}$ & 3B & 205.71 & 2.2 & Xgwm547 & 91.54 & 0.06 & O5 \\
\hline Yd_S1 & QYd_S1.NIAB-5Agg & $5 \mathrm{~A}$ & 158.81 & 2.09 & Xbarc165 & 99.37 & 0.07 & O5 \\
\hline RYd_S2 & QRYd_S2.NIAB-5A ${ }^{h h}$ & $5 \mathrm{~A}$ & 193.01 & 4.04 & $X g w m 1171 b$ & -7.68 & 0.3 & B2 \\
\hline YdD_S1 & $Q Y d D \_S 1 . N I A B-5 B^{l l}$ & $5 \mathrm{~B}$ & 143.21 & 2.66 & Xgwm1043 & 38.45 & 0.09 & O5 \\
\hline YdD_S1 & $\widetilde{Q} Y d D \_S 1 . N I A B-6 B$ & $6 \mathrm{~B}$ & 9.91 & 2.29 & Xgwm1199 & -35.55 & 0.08 & B2 \\
\hline RYd_S1 & QRYd_S1.NIAB-6B & $6 \mathrm{~B}$ & 121.71 & 2.09 & Xgwm963a & -3.85 & 0.07 & B2 \\
\hline Yd_S1 & QYd_S1.NIAB-7B & $7 \mathrm{~B}$ & 218.21 & 2.52 & Xbarc276 & -117.78 & 0.08 & B2 \\
\hline YdD_S2 & QYdD_S2.NIAB-7B & $7 \mathrm{~B}$ & 219.11 & 3 & Xbarc276 & -39.23 & 0.09 & B2 \\
\hline RHI_S2 & QRHI_S2.NIAB-1B $B^{b}$ & $1 \mathrm{~B}$ & 93.11 & 2.22 & Xgwm784 & 9.7 & 0.07 & O5 \\
\hline RHI_S2 & QRHI_S2.NIAB-2Bn & $2 B$ & 108.81 & 2.4 & Xgwm47 & -10.1 & 0.08 & B2 \\
\hline RHI_S2 & $\widetilde{Q R H I}$ S2.NIAB-3B.1 ${ }^{v}$ & 3B & 89.81 & 2.91 & Xbarc344 & -10.71 & 0.09 & B2 \\
\hline HI_S2 & QHI_S2.NIAB-3B ${ }^{x}$ & 3B & 205.71 & 2.42 & Xgwm547 & 2.03 & 0.08 & O5 \\
\hline RHI_S2 & QRHI_S2.NIAB-3B. $2^{x}$ & $3 B$ & 205.71 & 2.8 & Xgwm547 & -10.17 & 0.09 & B2 \\
\hline HID_S2 & QHID_S2.NIAB-4B & $4 \mathrm{~B}$ & 130.61 & 2.5 & Xbarc60 & 2.02 & 0.08 & O5 \\
\hline HID_S1 & QHID_S1.NIAB- $4 B^{c c}$ & $4 \mathrm{~B}$ & 151.21 & 2.59 & Xgwm940a & 3.31 & 0.09 & O5 \\
\hline HI_S1 & QHI_S1.NIAB-5B & $5 \mathrm{~B}$ & 228.61 & 2.77 & Xwmc28 & 3.19 & 0.1 & O5 \\
\hline HID_S2 & QHID_S2.NIAB-5B ${ }^{\text {ll }}$ & $5 B$ & 143.21 & 3.3 & Xgwm1043 & 2.24 & 0.11 & O5 \\
\hline HID_S2 & $\widetilde{Q} H I D \_S 2 . N I A B-7 A$ & $7 \mathrm{~A}$ & 108.61 & 2.42 & Xbarc108 & 1.8 & 0.07 & O5 \\
\hline RHI_S2 & QRHI_S2.NIAB-7Bzz & $7 \mathrm{~B}$ & 184.41 & 2.91 & Xgwm1085 & 11.25 & 0.09 & O5 \\
\hline HI_S1 & QHI_S1.NIAB-7B & $7 \mathrm{~B}$ & 336.01 & 2.42 & Xwmc273 & 2.95 & 0.08 & O5 \\
\hline \multicolumn{9}{|c|}{ Stress Indices } \\
\hline STI_S2 & QSTI_S2.NIAB-4A.1 & $4 \mathrm{~A}$ & 44.51 & 2.73 & Xbarc246 & 0.06 & 0.09 & O5 \\
\hline STI_S2 & QSTI_S2.NIAB-4A.2 & $4 \mathrm{~A}$ & 100.81 & 2.13 & Xgwm1234 & -0.06 & 0.06 & B2 \\
\hline STI_S1 & QSIT_S1.NIAB-6B ${ }^{S S}$ & $6 \mathrm{~B}$ & 159.91 & 2.14 & Xgwm889 & 0.06 & 0.07 & O5 \\
\hline STI_S2 & QSTI_S2.NIAB-7 $A^{t t}$ & $7 \mathrm{~A}$ & 0.01 & 3.36 & Xgwm1171a & -0.07 & 0.11 & B2 \\
\hline MP_S1 & QMP_S1.NIAB-1B & $1 \mathrm{~B}$ & 72.01 & 2.28 & Xgwm762 & 87.36 & 0.08 & O5 \\
\hline MP_S2 & QMP_S2.NIAB-1B & $1 \mathrm{~B}$ & 116.81 & 2.21 & Xbarc207 & -61.49 & 0.08 & B2 \\
\hline MP_S2 & $Q M P \_S 2 . N I A B-2 A^{j}$ & $2 \mathrm{~A}$ & 158.21 & 2.04 & Xgwm1256 & 97.14 & 0.22 & $\mathrm{O} 5$ \\
\hline MP_S2 & $\widetilde{Q M P} \bar{S} 2 . N I A B-3 B .1^{w}$ & $3 \mathrm{~B}$ & 194.81 & 2.87 & Xgwm 299 & 82.74 & 0.16 & O5 \\
\hline MP_S2 & $Q M P \_S 2 . N I A B-3 B .2^{x}$ & $3 B$ & 205.71 & 3.72 & Xgwm547 & 75.53 & 0.13 & O5 \\
\hline MP_S1 & QMP_S1.NIAB-5A ${ }^{g g}$ & $5 \mathrm{~A}$ & 158.81 & 2.4 & Xbarc165 & 63.78 & 0.08 & O5 \\
\hline ST_S2 & $Q S T \_S 2 . N I A B-3 B^{w}$ & 3B & 192.81 & 2.22 & Xgwm299 & 99.6 & 0.11 & O5 \\
\hline ST_S1 & QST_S1.NIAB-5A $A^{g g}$ & $5 \mathrm{~A}$ & 165.81 & 2.12 & Xbarc165 & 112.92 & 0.11 & O5 \\
\hline ST_S2 & QST_S2.NIAB-5A $A^{h h}$ & $5 \mathrm{~A}$ & 179.81 & 3.43 & Xgwm1171b & 116.02 & 0.13 & O5 \\
\hline DRI_S2 & QDRI_S2.NIAB-2B ${ }^{m}$ & $2 \mathrm{~B}$ & 52.21 & 2.02 & Xgwm972 & 59.7 & 0.06 & O5 \\
\hline DRI_S2 & QDRI_S2.NIAB-4A & $4 \mathrm{~A}$ & 64.31 & 2.47 & Xbarc343 & 82.63 & 0.1 & O5 \\
\hline DRI_S2 & QDRI_S2.NIAB-5A $A^{h h}$ & $5 \mathrm{~A}$ & 186.01 & 2.59 & $X g w m 1171 b$ & -81.06 & 0.11 & B2 \\
\hline DRI_S1 & QDRI_S1.NIAB-5Ahh & $5 \mathrm{~A}$ & 188.01 & 4.63 & Xgwm1171b & -111.01 & 0.26 & B2 \\
\hline DRI_S1 & QDRI_S1.NIAB-5B & $5 \mathrm{~B}$ & 232.71 & 2.44 & Xgwm1072 & 63.65 & 0.08 & O5 \\
\hline
\end{tabular}

\section{Discussion}

Climate change is causing more frequent and intense periods of drought as overall rainfall levels decline. Approximately $40 \%$ of the world's land surface is characterized as dry land which inhabits 2.5 billion people, which makes up 33\% of the global population [31]. Drought is currently one of the main constraints that prevent crop plants from expressing their full genetic potential. This makes the identification of drought-tolerant genotypes extremely important to secure productivity. In the present study, 114 RILs derived from a cross between Omrabi5 and Belikh2, where Omrabi5 was a drought tolerant but Bleikh2 a salt and high temperature tolerant cultivar were subjected to drought stress for two seasons to dissect drought tolerance in durum wheat. 
The genetic basis of most of the important traits in cereals is complex [32]. The same was observed in this investigation where the evaluation of 94 parameters resulted in the discovery of 221 QTLs distributed all over the durum wheat genome (Table 3). There were thirteen traits (Hd_S2, RHd_S2, TrD_S2, RSc_S1, PhrD_S1, RPhr_S1, RWue_S1, RWue_S2, RS1_S2, Sw_S2, RHI_S1, SSI_S1 and SSI_S2) for which no QTL was discovered, possibly because of the limitation of bi-parental population which is derived from two parents following fewer recombination events.

Table 3. Percentage increase or decrease in yield under various conditions with respect to Xgwm895 at $2 \mathrm{~A}$ and $\mathrm{Xbarc} 276$ at $7 \mathrm{~B}$.

\begin{tabular}{cccccccc}
\hline Trait & \multicolumn{2}{c}{ Xgwm895, 2A } & \multicolumn{2}{c}{ Xbarc276, 7B } & \multicolumn{3}{c}{ Combination } \\
\hline & A allele & B allele & A allele & B allele & AA & BB & AB \\
\hline Yd_S1 & 5.5 & -9.31 & 5.5 & 6.6 & -4 & -1.89 & 2.16 \\
YdD_S1 & 0.18 & -1.6 & 0.18 & 0.66 & 0.61 & -1.83 & -0.15 \\
RYd_S1 & -6.65 & 6.82 & -6.65 & -4.66 & 1.56 & 2.26 & -14.1 \\
Yd_S2 & 6.16 & -9.35 & 6.16 & 2.57 & 0.92 & -10.78 & 10.2 \\
YdD_S2 & 5.27 & -8.4 & 5.27 & 4.39 & 22.98 & -10.43 & 11.3 \\
RYd_S2 & -1.59 & 1.85 & -1.59 & 2.61 & -3.04 & 4.74 & -0.39 \\
\hline
\end{tabular}

\subsection{Phenotypic Differences between Treatments and Seasons}

The different phenotypic responses to drought treatment and seasons within our RILs empowered us to genetically dissect their performance through QTL analysis. From yield perspective, yield was considerably reduced in drought in both S1 and S2. However, reduction was slightly more pronounced in S2 (RYd_S2 $=34.60 \pm 12.27)$ as compared to S1 $\left(\mathrm{RYd} \_\mathrm{S} 1=36.39 \pm 12.21\right)$. This superior yield in S1 could be attributed to $\mathrm{Sl}, \mathrm{Sw}$ and $\mathrm{Sps}$, as these traits were higher in drought treatments in $\mathrm{S} 1$ than in the same treatment in S2. Seasonal difference might also be due to change in mean minimum and maximum temperatures during the two seasons as the mean minimum temperature in $\mathrm{S} 1\left(16.81^{\circ} \mathrm{C}\right)$ was $1.24^{\circ} \mathrm{C}$ less than mean minimum temperature in S2 $\left(17.57^{\circ} \mathrm{C}\right)$ although there was almost no difference on average temperatures during S1 and S2 (Table S6). Besides, the relatively improved performance in drought in S1 of RILs could also be ascribed to $11 \mathrm{~mm}$ rain received in the month of Jan (when plants are heading which is sensitive to abiotic stress [33]) in S1. Superior performance in control over treatment in both S1 and S2 could also be due to prolonged growing season ( $\sim$ week) beside other advantages as treatment caused early flowering that reduced the assimilates time to move to plant development and growth [34].

\subsection{Agronomic Traits}

Germination plays an important role in the plant stand, which guarantees a successful harvest. In this study, the markers linked to QGr_S1.NIAB-5A.1 (Xbarc342) and QGr_S1.NIAB-6B.1 (Xbarc178) have been reported to be linked with initial and relative germinations, respectively $[35,36]$, has reported one Hd QTL on the same chromosome at $58.2 \pm 38.6 \mathrm{cM}$. Therefore, it can be concluded that the same QTL may be present in our study influencing Gr. Another Gr QTL (QGr_S1.NIAB.5A.2) is located with several other QTLs linked with Hd, Yd, ST and DRI. Six QTLs of Gr on chromosome 5B indicating that this is an important site for genes linked to plant survival.

As regards the heading date, [29] discovered an Hd QTL at $40 \mathrm{cM}$ on chromosome 2A in a durum wheat population whereas the QTL QHdD_S1.NIAB.2A in our present study was at $5 \mathrm{cM}$ on chromosome 2A. The QTL QHd_S1.NIAB-3A is highly comparable with the one detected by [35] for relative germination. Moreover, it is also the site of many other QTLs linked to spike related traits in this study indicating that plant development influences spike related traits and in turn final yield. It should be mentioned that a flowering time locus has been reported by [37] on chromosome 3AL. Another QTL QHd_S1.NIAB-5A is linked to Yd, MP and ST QTLs in this study. In addition, this QTL and QHdD_S2.NIAB-5A are in close vicinity to each other. Thus, the Hd QTL on chromosome 5A 
can be a constitutive QTL whose selection may help in yield increase as QHdD_S2.NIAB-5A is also linked to Yd, ST and DRI QTL in this investigation. Two Hd QTLs on chromosome 5A have been reported by [32]. Moreover, [37] discovered an Hd QTL on chromosome 5A, affirming the finding of this study. On chromosome 7B, [29] have located an Hd QTL between 0 and $44.3 \mathrm{cM}$ whereas our QTL (Q.RHd_S1.NIAB-7B) is located at $42.5 \mathrm{cM}$ confirming the findings of [29].

Most of the Ph QTLs were concentrated on chromosomes 4B and 5A. Many Ph QTLs have been reported to be located on chromosomes $1 \mathrm{~A}, 1 \mathrm{~B}, 1 \mathrm{D}, 2 \mathrm{D}, 3 \mathrm{~A}, 4 \mathrm{~A}, 4 \mathrm{~B}, 6 \mathrm{~A}, 7 \mathrm{~A}$ and $7 \mathrm{~B}[32,38,39]$. The marker linked to QPhD_S2.NIAB-4B and QRPh_S2.NIAB-4B (Xbarc193) has been reported to be linked with coleoptile length by [40]. The same area has also been reported to carry Ph QTLs in durum wheat [39]. Likewise QPh_S1.NIAB-6A is highly comparable with the QTL discovered by [32] on chromosome 6A. Ref. [38] reported a QTL for Ph under drought conditions between 38 and $50 \mathrm{cM}$ and another QTL under well-watered conditions at the distal end of chromosome 7AL. In addition, another QTL for Ph under drought stress has been reported to be on chromosome 7B by [38], which is linked to marker Xbarc1073. Our QTL QPhD_S2.NIAB-7A linked to marker Xwmc517 can be highly comparable to the one reported by [38] because the marker Xwmc517 is closely linked to Xbarc1073 in [38]. Several Ph QTLs were co-located with QTLs of other traits. For example, QPhD_S2.NIAB-5A was co-located with Sc, Tr, Wue, Sw and Tkw loci. To add to this, QRPh_S1.NIAB-7B was co-located with a Tr QTL.

\subsection{Physiological Traits}

In our study, the QTL QTrD_S1.NIAB-2B for Tr may be the same as Qgs-2B.1 (for Tr) by [38], as both lie on the proximal ends of chromosome 2BS. Another marker (Xwmc50) of QRTr_S1.NIAB-3A is reported to be linked with relative germination after experimental ageing [35] indicating the presence of stress resistance genes in this area. Ref. [38] located a QTL linked to Tr under irrigated conditions at the distal end of chromosome 5AS, whereas the QTL QTr_S2.NIAB-5A in our investigation was at the far end of chromosome 5AL, indicating that QTr_S2.NIAB-5A is a novel QTL. Similarly, the two QTLs (QTr_S2.NIAB.5B and QRTr_S2.NIAB.5B) at $158 \mathrm{cM}$ linked to marker Xgwm408 are highly comparable to one identified by [37] where the authors located a QTL linked to transpiration rate at early grain filling stage under irrigated conditions. Ref. [35] also located a $\operatorname{Tr}$ QTL in the distal region of chromosome 5AL. The QTL QTrD_S1.NIAB.7B.2 is connected to marker Xbarc276, which is the same marker reported by [37] to be linked with Tr under irrigated conditions on chromosome 7B.

In case of Sc, the QTL QScD_S2.NIAB.2B at $107.81 \mathrm{cM}$ on 2B may be the same as observed by [38] at $\sim 95 \mathrm{cM}$ under drought stress. Ref. [37] detected several QTLs linked to leaf porosity (LP) on the distal end of chromosome 3BS. Our QTL QSCD_S2.NIAB-3B at $13.91 \mathrm{cM}$ on 3B might correspond to any of the LP QTLs of [37].

The 17 QTLs linked to Phr are distributed on all chromosomes except 1A, 4A and 5A, and most of them were discovered in S2 only. Nevertheless, QPhrD_S2.NIAB-3B.1 and QPhrD_S2.NIAB-3B.2 mirrored to a photosynthesis QTL identified by [37] under drought stress. The linked marker in both studies was Xgwm299. [35] located one Phr QTL under drought in the distal region of chromosome 3BS. In addition, [37] also located another Phr QTL under drought stress in the centromere region of chromosome 2B whereas in our study QRPhr_S2.NIAB.2B was located at the distal end of chromosome 2B. One drought and one well-watered Phr QTL by [35] and one LP QTL by [37] was located on chromosome 4B. Whether QPhr_S1.NIAB-4B matches any of them can only be speculated at this stage. To add to it, like in this study, [35] located Phr QTL on chromosomes 6B and 7A. However, they were at different positions. The QTL QPhrD_S2.NIAB-7B co-locates with normal seedling and total germination QTL of [40]. Interestingly, another QTL on 7B (QRPhr_S2.NIAB-7B) co-locates with Tkw, seed area and seed length by [40]. Furthermore, this QTL can also be mirrored with Phr QTLs by [41].

In the case of Wue, [38] located Wue QTLs at $94 \mathrm{cM}$ on 2B and at $\sim 238 \mathrm{cM}$ on $7 \mathrm{~A}$, whereas QWueD_S1.NIAB-2B and QWue_S1.NIAB-7A in our study were located at the very distal ends of 2BS and 7AS, respectively, and hence are not comparable to the ones detected by [38]. However, the QTL QWueD_S2.NIAB-7B co-located with QRPhr_S2.NIAB-7B mirrored with Phr QTLs by [41]. 


\subsection{Spike-Related Traits}

With respect to Sl, QSl_S1.NIAB-2A mirrors with a grain protein content (GPC) QTL in [42], whereas QSID_S1.NIAB-2B mirrors with Sps QTL in [43]. This QTL, however, is not the same as that described by [44], who detected consistent large effect QTLs for $\mathrm{Sl}$ in six environments. To add to this, Xwmc50 and Xgwm940a of QTLs QRSI_S1.NIAB-3A and QSID_S2.NIAB-4B, respectively, showed connections with germination under normal and stress conditions [35]. The QTL QSL_S2.NIAB-5B is also at the location of numerous QTLs linked with yield related traits in [45]. Similarly, a QTL linked to $\mathrm{Sl}$ has been reported by [45] on the site of QSID_S2.NIAB-7A. Ref. [38] also reported a QTL linked to $\mathrm{Sl}$ under drought stress at $160 \mathrm{cM}$ on chromosome 7A. Furthermore, two QTLs at $\sim 73 \mathrm{cM}$ and $148 \mathrm{cM}$ have been reported to be linked with $\mathrm{Sl}$ in irrigated and drought conditions, respectively. We speculate the 7B QTLs of $\mathrm{Sl}$ (QRSI_S1.NIAB-7B and QRSI_S2.NIAB-7B) might correspond to any of those mentioned in [38]. The site of QTLs QSwD_S1.NIAB-1A and QSw_S1.NIAB-1A is reported to carry Sps QTLs [38]. Likewise, the QTLs $Q S w D \_S 1 . N I A B-2 A$ and $Q R S w \_S 1 . N I A B-2 A$ are in the region of GPC QTLs [42]. The QTLs on 3A (QSwD_S1.NIAB-3A and QRSw_S1.NIAB-3A) can be compared with relative germination QTLs [35] and Sw QTLs in [45]. The site of QTL QSwD_S1.NIAB-3B has been reported for SI QTL in different environments [43]. Likewise, other QTLs have been known to be in the region of QTLs linked to yield and related traits [38].

Many QTLs linked to yield and spike traits have been mapped to chromosomes where Sps QTLs of this study are mapped $[32,37,43]$. In particular, the marker Xwmc218 of QSpsD_S2.NIAB-7B has been reported to be in connection with seed area and length in [40], providing a clear connection towards yield. Moreover, QRSps_S1.NIAB in this study is located at $104 \mathrm{cM}$ mirrored with grains per spike QTL of [44] who reported the Sps QTL at $102.5 \mathrm{cM}$.

Four QTLs among 16 of Tkw were co-located with other Tkw QTLs. The 1B loci (QRTkw_S.NIAB-1B, QTkwD_S1.NIAB-1B.1 and QTkw_S1.NIAB.2) can be compared with the Yd QTLs of [38] and meta-QTL of Yd by [43]. Furthermore, QRTkw_S.NIAB-1B, QTkw_S2.NIAB-4B, QRTkw_S2.NIAB-4B, QTkDw_S1.NIAB-5A, QRTkw_S1.NIAB.5A and QRTkw_S1.NIAB.7B mirror relative germination QTLs by [35].

\subsection{Yield and Related Traits}

Chromosome 1B is an important site of yield QTLs as several yield QTLs on this chromosome in hexaploid [29] or durum wheat [38,46]. Thus, the QTL QRYd_S2.NIAB-1B could be one reported previously. Interestingly, this QTL also co-locates with coleoptile length under drought [40] in the same population, pointing towards stress tolerance genes in this area. Similarly, there were three QTLs for yield ( $Q Y d D \_S 1 . N I A B-2 A, Q Y d D \_S 2 . N I A B-2 A$ and $\left.Q R Y d \_S 1 . N I A B-2 A\right)$ on chromosome 2A where QYdD_S2.NIAB-2A and QRYd_S1.NIAB-2A were linked to the same marker (Xgwm895). Consistent QTLs for Yd have been detected on chromosome 2A [43,45]. The QTL QYdD_S1.NIAB-2A can be compared with 2AS Yd QTLs of [42], whereas the other two can be compared with the QTLs on 2AL. Ref. [29] also detected a Yd QTL on 2A linked to marker Xwmc177, which is closely linked to Xgwm726 (related to QYdD_S1.NIAB-2A). The 3A QTL (QYd_S2.NIAB-3A) seems not to be comparable to those observed by [29] in durum wheat and by [36] in hexaploid wheat, because their QTLs were either in the centromere region or at the distal end of 3AL whereas our QTL was at $52.11 \mathrm{cM}$. However, this QTL could be compared Yd QTL described by [34], who described a cluster of QTLs including Yd QTL between 41.3 and $51.4 \mathrm{cM}$ in durum wheat. Moreover, [46] have also located a Yd QTL under drought stress at $62.9 \mathrm{cM}$. To add to it, QYd_S2.NIAB-3A does co-locate with a grain number QTL of [32]. The QTLs viz. QYd_S2.NIAB-3B and QRYd.NIAB-3B linked to marker Xgwm547 are at the tip of long arm of 3B. Consistent QTLs for Yd under heat, drought and irrigated conditions have been described by [34] at $212 \mathrm{cM}$ on chromosome 3BL indicating that our QTL could be the same as that of [34]. Likewise, both [29] and [45] have located QTLs for Yd at the same location, whereas [36] located a Tkw QTL in hexaploid wheat in this area indicating the connection of Yd with Tkw. One of the two 5A QTLs (QYd_S1.NIAB-5A and QRYd_S2.NIAB-5A) co-locates with a germination QTL in [35], 
whereas [32] located several Sl, Sw and Tkw QTL on the entire length of chromosome 5A, suggesting some connection between yield and these components. In addition, [32] have reported numerous QTLs linked to yield and related traits on chromosome 5A [47]. Furthermore, both [32] and [47] have reported Yd QTLs on the proximal end of chromosome 5BS, whereas QYdD_S1.NIAB.5B lies at $143.21 \mathrm{cM}$, and so cannot be compared with either of the above-mentioned investigations. Ref. [29] located a yield locus exactly in the same area as that of QRYd_S1.NIAB.6B. Nevertheless, [32] mapped numerous Yd related QTL on chromosome 6B. Chromosome 7B has been reported to be an important site for $Y d$ and $Y d$ related traits by [32], and [29] located a stable QTL for Yd between 19 and $33 \mathrm{cM}$ and one QTL restricted to one environment between 106 and $122 \mathrm{cM}$. This study linked QYd_S1.NIAB.7B and QYdD_S2.NIAB.7B (216-226 cM) with Xbarc276, which is in between Xgwm333 and Xbarc176. This interval is linked with a Yd QTL in [29].

There are no investigations exclusively reporting Bm QTLs except [38] and [48]. No QTLs of Bm in the present study match with those reported by [38] and [48]. However, the QTL QBmD_S1.NIAB-7A of 7A is very near to GPC and SI QTL as reported by [32] in hexaploid wheat. Likewise, the QRBm_S2.NIAB-7B of 7B is linked with QTLs of seed area and seed length [41]. Among other traits, the Bm QTLs co-locate with Gr, Ph, HI, Sw, Sps, Yd and HI QTLs in this population indicating that these are the sites of important genes.

The importance of $\mathrm{HI}$ in relation to drought has been indicated recently [36,44]. None of the $12 \mathrm{HI}$ QTLs in this study matched with those of [38], who located HI QTLs in hexaploid wheat on chromosomes 1A, 2A, 2B, 3A, 4A, 4D and 6A. Numerous QTLs for HI have been identified on chromosomes 1B, 5B, 5D 6A, 7A, 7B and 7D using an association mapping approach and a different marker system [49]. The 1B QTL of HI (QRHI_S1.NIAB-1B) in this study was located at $93.11 \mathrm{cM}$ whereas the QTL in [49] was located at $158 \mathrm{cM}$; hence, they seem not to be comparable with each other. QRHI_S1.NIAB-1B also co-locates with coleoptile length under drought stress in [41]. The QTL QHID_S.NIAB-5B on 5B can be the same as that of [49] who discovered an HI QTL under non stress conditions. The chromosome 7B carried QTLs for HI in non-stress, drought, heat and combined heat and drought stress most notable at $163 \mathrm{cM}$ in [49], whereas our QTLs QRHI_S2.NIAB-7B and QHI_S2.NIAB-7B were at a distance of 184.41 and $336.01 \mathrm{cM}$, respectively.

\subsection{Stress Indices}

Several tolerance indices have been devised and used by breeders to identify the best droughttolerant lines. It has been suggested that each index may be a potential indicator of different biological responses to drought [31]. STI, MP and geometric mean productivity have been concluded to be effective indices in discriminating drought tolerance genotypes in durum wheat [50]. Chromosome 2B has been an important site harboring STI QTLs under drought, heat and combined drought and heat stress [49]. However, our QTL QDRI_S2.NIAB-2B does not correspond to any of those QTLs because of a different position. Chromosome $4 \mathrm{~A}$ also carried STI QTLs under combined stress at 67.71 and $71.47 \mathrm{cM}$ in [49] indicating some similarity with our 4A QTLs which were at 44.51 (QSTI_S2.NIAB-4A.1), 64.31 (QDRI_S1.NIAB-4A) and 100.81 (QSTI_S2.NIAB-4A.2) cM. To add to it, [50] also located various QTLs linked with yield and yield related stress indices on chromosome 4A. The marker Xgwm 299 on 3B in association with QMP_S2.NIAB-3B.1 and QST_S2.NIAB-3B co-locates with Phr, Sw and Sps QTLs. Furthermore, the same marker is linked with Tkw [51] and Phr at late growing stages [40] in other studies. Furthermore, the interval of QDRI_S1.NIAB-5B (Xwmc28-Xgwm1072) is linked with HI, spike $\mathrm{HI}$ and spike number QTLs in durum wheat [52].

\subsection{Co-Location QTLs and MAS for Durum Wheat Breeding}

From the total of 221 QTLs, 145 could be clustered (2 or more QTLs linked to the same marker) into a total of 53 clusters (Figure 1), covering all the durum wheat chromosomes except $4 \mathrm{~A}$. The number of QTLs clustered together ranged between two and six. Among them, there were at least 20 markers that were associated with any trait in both seasons, whereas 10 markers were associated with the 
same trait under various conditions in a single season. Moreover, there were four markers that were associated with the same trait under different conditions in both seasons. It is also evident that certain Yd QTLs match exactly with Gr (cluster 5A.5), Tr (cluster 7B.5), Hd (cluster 5A.4, 5A.5), Tkw (clusters 1B.1, 2A.1), HI (cluster 3B.5), Sps (cluster 5B.2) and stress indices (clusters 5A.4, 5A.5) QTLs. Clusters of QTLs have also been reported previously $[32,34,38,45,47]$, indicating that multiple genes might be present at such sites which can either be constitutive or expressive. Plant breeders, however, tend to look for stable loci to improve yield. Although several QTLs can be targeted for yield enhancement, the most promising one could be the clusters 2A.4 and 7B.5, where markers Xgwm895 and Xbarc276 were associated with $\mathrm{Yd}$ in both S1 and S2. The allelic profile of yield QTLs in these clusters indicate that allele A of Xgwm895 and allele B of Xbarc276 can enhance the Yd up to $6.16 \%$ in control and $5.27 \%$ in drought (Table 4). Moreover, if combined, a yield gain of up to $11 \%$ is possible.

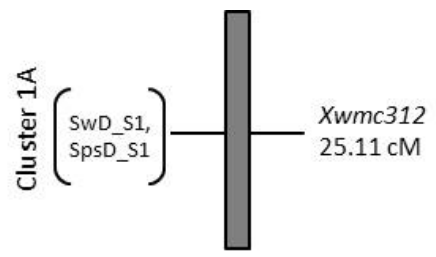

$1 \mathrm{~A}$

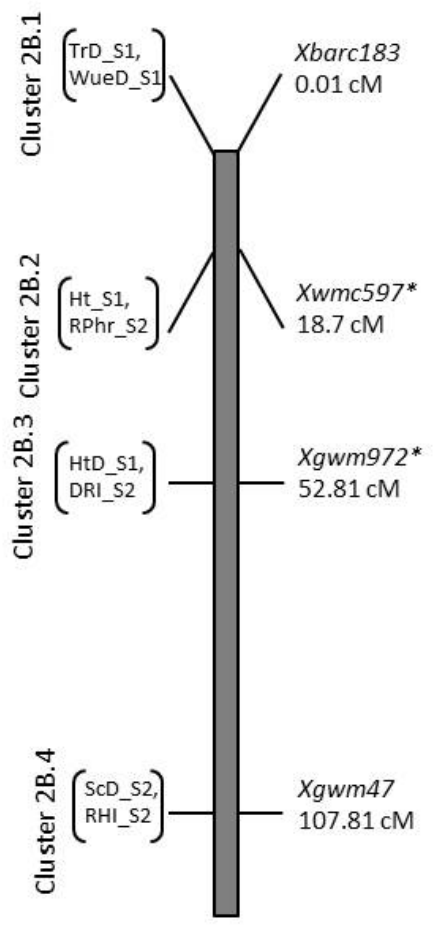

2B
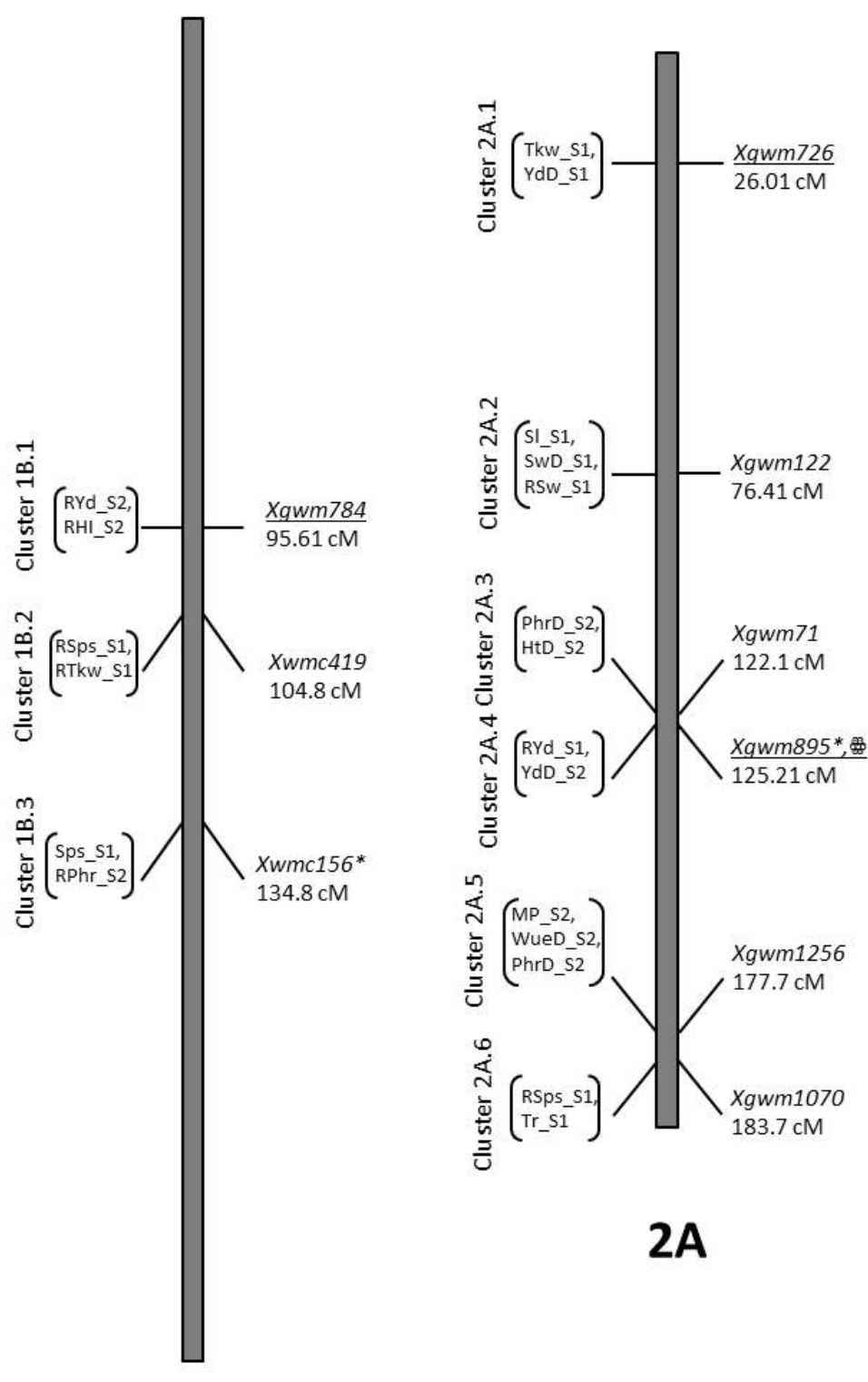

$2 A$

\section{B}

Figure 1. Cont. 


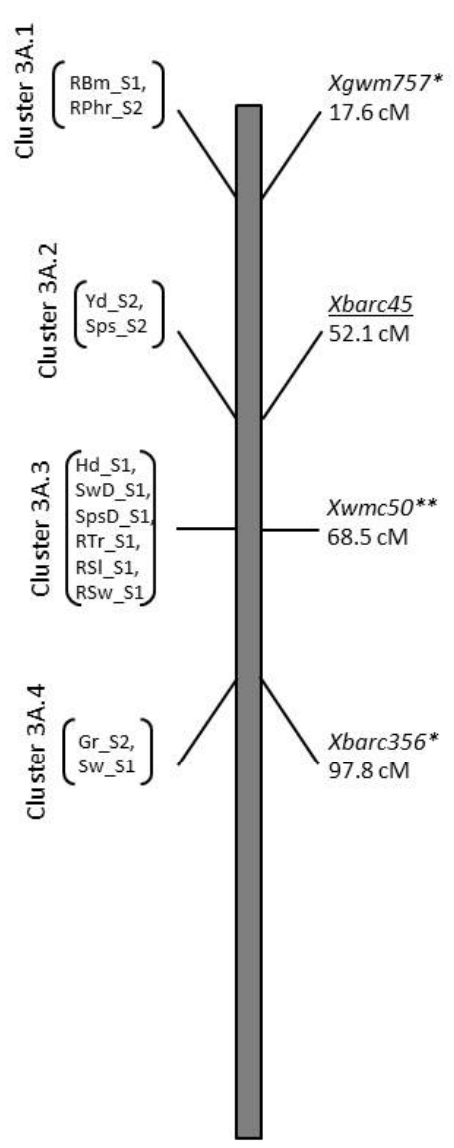

3A
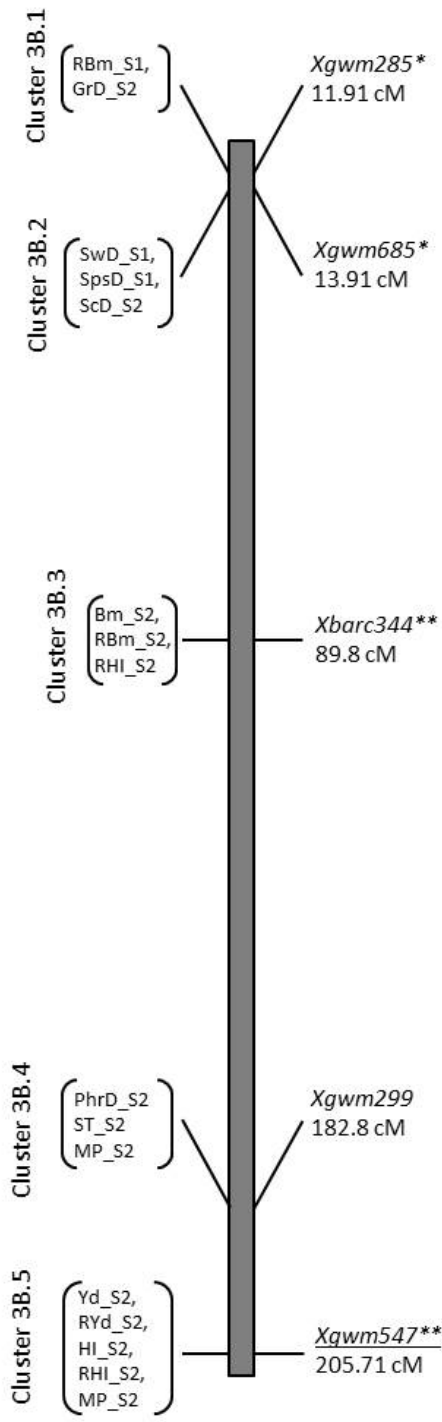

3B

Figure 1. Cont.

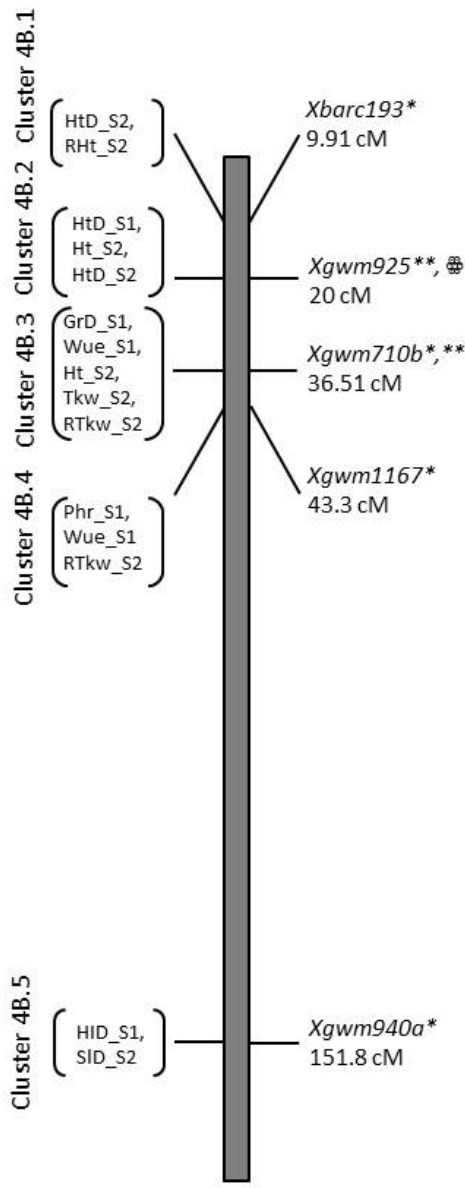

4B 


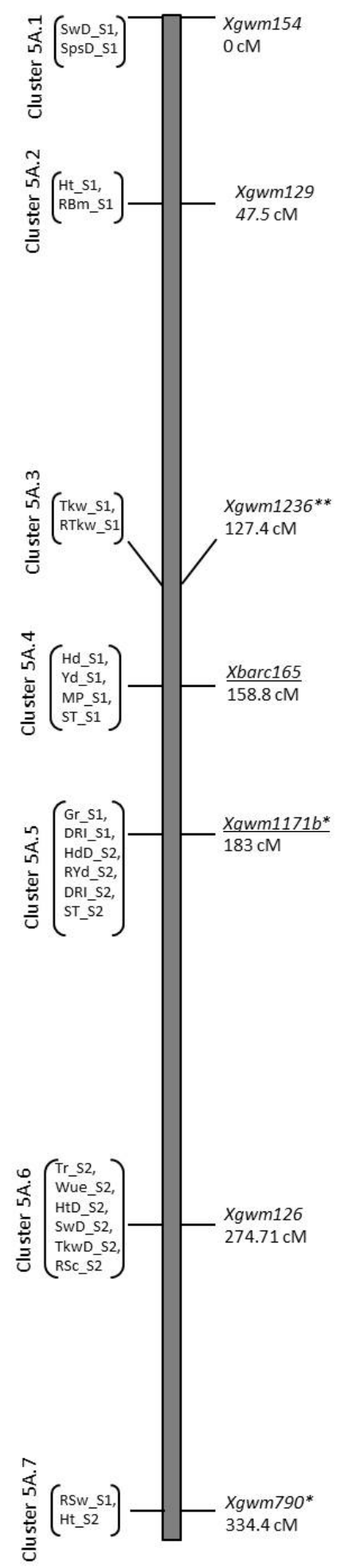

5A

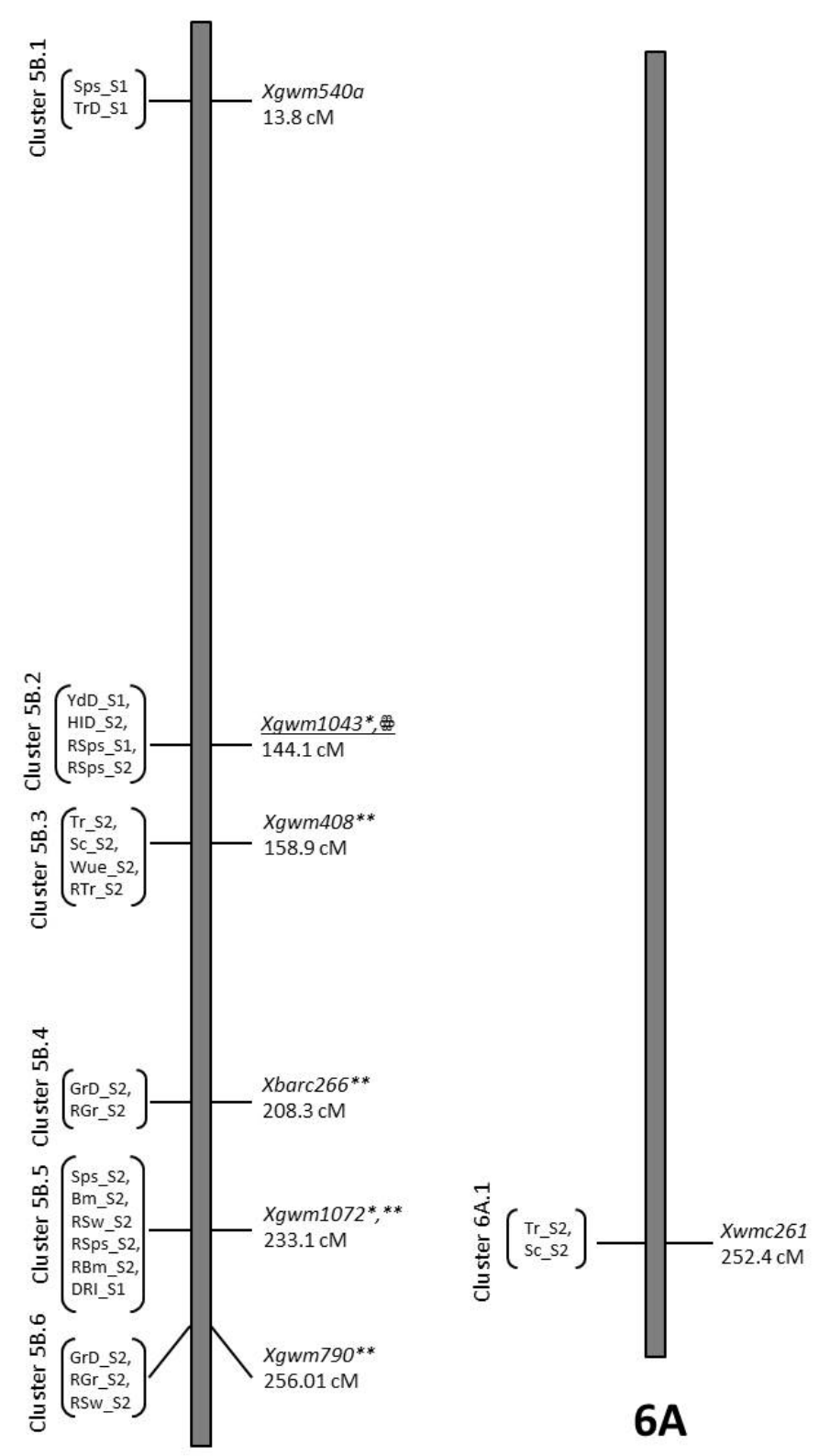

5B

Figure 1. Cont. 

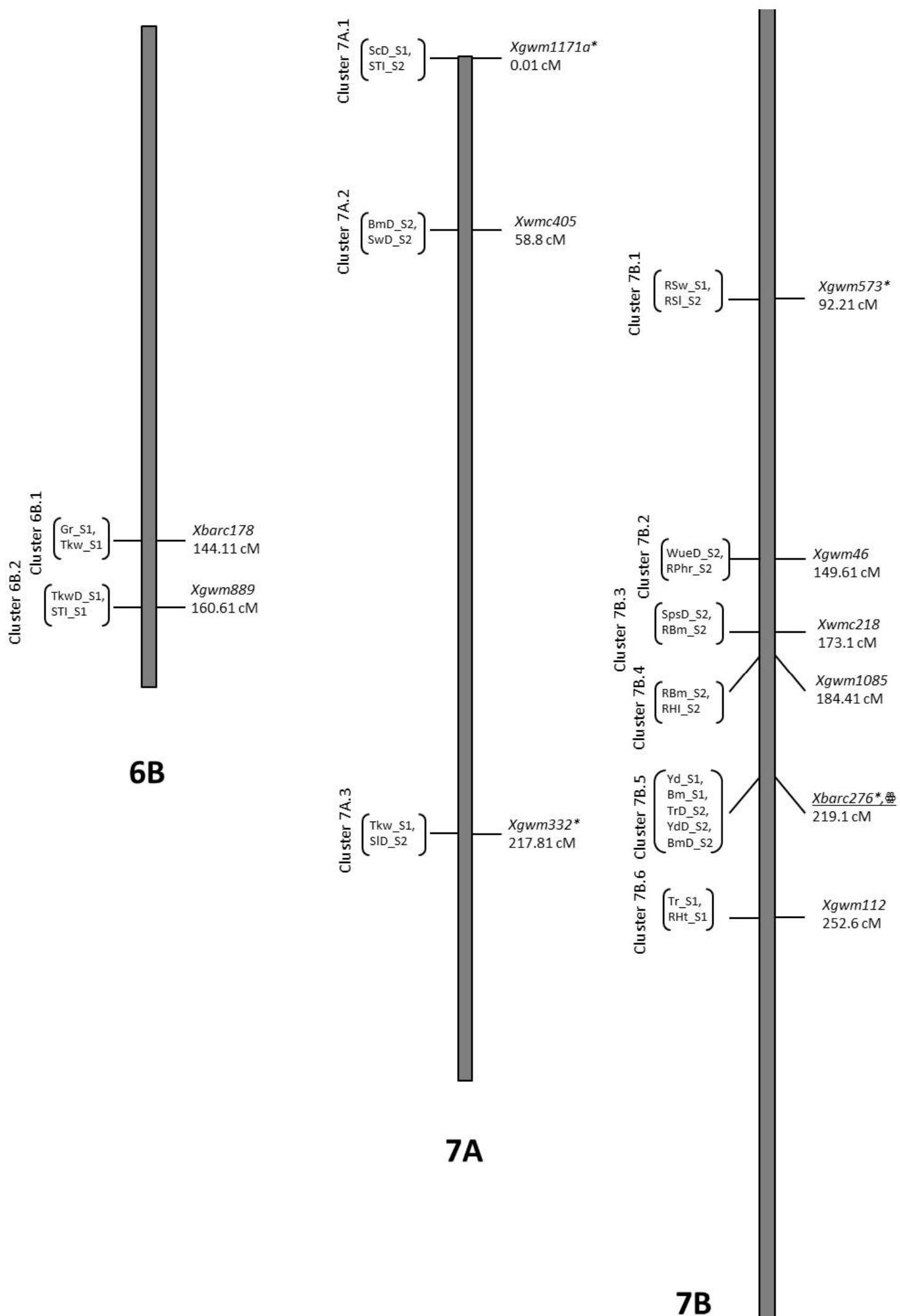

\section{B}

\section{A}

7B

Figure 1. Distribution of QTL clusters in RIL population of durum wheat population. ${ }^{*}=$ marker linked with a QTL in both seasons for any trait, ${ }^{* *}=$ marker linked with the same trait in different conditions in a single season, both seasons. Markers linked with any yield QTL are under lined. For abbreviation, refer to Table 4. 


\section{Materials and Methods}

A set of 114 RILs was developed from a cross between a drought-tolerant cultivar Omrabi5 (P1) and a high temperature- and salinity-tolerant breeding line Belikh2 (P2) [17] at ICARDA, Syria, by repeated selfing of $F_{1}$, using the single seed descent method [28]. Seeds from the 2015 season in Germany were multiplied in 2015-16 at NIAB, Pakistan, and were subsequently used for the investigation in 2016-17 and the seeds of the 2016-17 harvest were used in 2017-18.

\subsection{Experimental Design and Traits Measured}

A total of two experiments were conducted to investigate the population with respect to yield and other related traits. They were conducted in 2016-17 (S1) and 2017-18 (S2) seasons at NIAB, Pakistan, at exactly the same experimental site to avoid any soil interaction. Moreover, all the data were collected at almost the same dates in both experiments to minimize the effect of environment on phenotypic expression of the measured traits. The population was sown on 1st of November each season with one replicate in control and three under drought in both S1 and S2 cycles in the form of two rows per RIL. Seeds were sown manually with $5 \mathrm{~g}$ seeds/row, in 2-meter rows using hand-driven drill and row to row distance was kept $20 \mathrm{~cm}$. Control plots were irrigated four times (15th Nov., 1st Jan., 15th Feb. and 15th March), whereas drought plots were kept devoid of any irrigation. The drought and control experiments were in the same field, where the control experiment was separated from the drought by making a 2 -ft-high ridge partition and there was a gap of at least 5 meters between drought and control treatments. Furthermore, there was also a gap of 5 meters between drought treatment and water channel to avoid any leach down effect that might have an effect on drought treatments. To add to it, we planted brassica in the leftover area so that the leached water from the water channel and control experiment is used up before it reached to the drought treatment. The total rainfall in S1 was 36.25 days, distributed over the period of 14 days (from Nov 2016-May 2017), where the maximum rain was received in April 2017 (16.58 mm). In S2, total rainfall was 42.48, distributed over a period of 30 days with maximum rainfall in April 2018 (Table S6). Furthermore, temperature data is provided in Table S7 and layout plan of the experiments is provided in Figure S1. A list of parameters recorded during the course of the two experiments is given in Table 4. Correspondingly, relative values were also calculated by dividing drought by the control times one hundred.

\subsubsection{Agronomic Traits}

Germination \% $(\mathrm{Gr} / \mathrm{GrD})$ was recorded visually three weeks after sowing according to a scale ranging from $0 \%-100 \%$, where $0 \%=$ no germination and $100 \%=$ complete germination. Heading time $(\mathrm{Hd} / \mathrm{HdD})$ was measured when $50 \%$ of spikes have left the flag leaf. Plant height $(\mathrm{Ph} / \mathrm{PhD})$ was recorded in $\mathrm{cm}$ from five random plants per replicate, with the help of meter rod, measured from the base of the plant to the spike tip excluding awns, before harvest.

\subsubsection{Physiological Traits}

Transpiration rate $(\operatorname{Tr} / \operatorname{TrD})$ was calculated using the handheld porometer (Model: LI-1600 steady state porometer) in $\mu \mathrm{g} \mathrm{cm}^{-2} \mathrm{~s}^{-1}$. Data were recorded separately on cloudless days for control (on 99th day of sowing) and drought (on 100th and 101st day of sowing) between 11:00 am and 2:00 pm. Each reading was a mean of three randomly selected flag leaves per replicate. Stomatal conductance $(\mathrm{Sc} / \mathrm{ScD})\left(\mathrm{mmol} \mathrm{m}^{-2} \mathrm{~s}^{-1}\right)$ was calculated using the formula: $1 / \mathrm{DR} \times \mathrm{CF}$ where $\mathrm{DR}$ is diffusible resistance calculated from porometer, $\mathrm{CF}=$ correction factor which is calculated using the formula: $\mathrm{LT} \times$ constant where the value of constant at $25^{\circ} \mathrm{C}$ for durum was 411.8 , and LT was leaf temperature calculated from porometer. Photosynthetic rate $(\mathrm{Phr} / \mathrm{PhrD})$ was determined from Sc and $\mathrm{Tr}$ using the formula: $\mathrm{Phr}=\mathrm{Sc}$ $\left(\mathrm{mmol} \mathrm{m} \mathrm{s}^{-2} \mathrm{~s}^{-} \operatorname{Tr}\left(\mu \mathrm{g} \mathrm{cm}^{-2} \mathrm{~s}^{-1}\right) \times 10\right.$. Water use efficiency (Wue/WueD) was calculated by dividing the corresponding photosynthetic rate with transpiration rate times 100. 


\subsubsection{Spike-Related Traits}

For spike-related traits, five spikes per row per replicate were collected randomly from the two experiments whereas for other traits, values from random plants were taken. Spike length (Sl/SID) was calculated by taking mean length of five spikes/replicate using scale in centimeters. Spike weight (Sw/SwD) in grams was calculated by taking mean weight of five spikes using electronic weighing balance. The number of seed per spike (Sps/SpsD) was calculated by threshing and counting the seeds from five random spikes per replicate. Thousand kernel weight $(\mathrm{Tkw} / \mathrm{TkwD})$ in grams was recorded by weighing 1000 grains using electronic balance.

\subsubsection{Yield and Related Traits}

These included biomass (Bm), yield (Yd) and harvest index (HI). Biomass (Bm/BmD) $(\mathrm{g})$ was measured by weighing the complete harvest of above ground part excluding roots per plot where harvesting was done manually to avoid any mixing of the straw. Yield (Yd/YdD) was recorded after manual threshing in grams per plot. Harvest index (HI/HID) was determined by dividing the corresponding $\mathrm{Yd}$ with respective Bm.

\subsubsection{Stress Indices}

Five stress indices were calculated using the yield data. These included stress tolerance index (STI), mean productivity (MP), stress tolerance (ST), stress susceptibility index (SSI), and drought resistance Index (DI), whose formulas are provided in Table 4.

\subsection{Data Analysis}

Statistica software was used for descriptive statistics of the measured traits and to construct box plots. ANOVA was performed using the RStudio-1.0.153.exe program.

\subsection{Genotyping and Genetic Mapping}

The genetic map of Omrabi $5 \times$ Belikh2 RIL population comprising 265 microsatellite loci, spanning $2864 \mathrm{cM}$ (10.8 cM mean inter-marker separation) and covering all the 14 chromosomes of durum wheat, was available from [40]. The individual chromosomes ranged in genetic length from $37 \mathrm{cM}$ (chromosome 1A) to $366 \mathrm{cM}$ (chromosome 7B). The 265 microsatellites comprised 159 GWM, 62 BARC and 44 WMC markers. Further details about the map are available in [40]. QTLs were assigned using a composite interval mapping method (model 6 with forward stepwise regression) implemented in QTL Cartographer v2.5 [53]. To control the effect of genetic background, five markers, identified by forward regression, were used as a cofactor with a window size of $5 \mathrm{cM}$. A LOD score of $>2.0 \leq 3$ was applied to detect QTLs as significant and $>3.0$ as highly significant.

Table 4. Absolute and relative traits measured for RIL population in 2016-17 (trait abbreviation followed by _S1) and 2017-18 (trait abbreviation followed by _S2) in control and drought and their time of measurement each season.

\begin{tabular}{|c|c|c|c|c|c|}
\hline & Traits & Control & Drought & Relative & Time \\
\hline \multicolumn{6}{|c|}{ Agronomic Traits } \\
\hline 1 & Germination & Gr_S1, Gr_S2 & GrD_S1,GrD_S2 & RGr_S1, RGr_S2 & 24th Nov \\
\hline 3 & Plant height & Ph_S1, Ph_S2 & PhD_S1, PhD_S2 & RPh_S1, RPh_S2 & Before harvest \\
\hline \multicolumn{6}{|c|}{ Physiological Traits } \\
\hline 5 & Stomatal conductance & Sc_S1, Sc_S2 & ScD_S1, ScD_S2 & RSc_S1, RSc_S2 & $7^{\text {th }}$ Feb \\
\hline 6 & Photosynthetic rate & Phr_S1, Phr_S2 & PhrD_S1, PhrD_S2 & RPhr_S1, RPhr_S2 & $7^{\text {th }} \mathrm{Feb}$ \\
\hline 7 & Water use efficiency & Wue_S1, Wue_S2 & WueD_S1, WueD_S2 & RWue_S1, RWue_S2 & $7^{\text {th }}$ Feb \\
\hline
\end{tabular}


Table 4. Cont.

\begin{tabular}{|c|c|c|c|c|c|}
\hline & Traits & Control & Drought & Relative & Time \\
\hline \multicolumn{6}{|c|}{ Spike-Related Traits } \\
\hline 8 & Spike length & S1_S1,S1_S2 & S1D_S1, S1D_S2 & RS1_S1, RS1_S2 & After harvest \\
\hline 9 & Spike weight & Sw_S1,Sw_S2 & SwD_S1,SwD_S2 & RSw_S1, RSw_S2 & After harvest \\
\hline 10 & Seeds per spike & Sps_S1, Sps_S2 & SpsD_S1,SpsD_S2 & RSps_S1, RSps_S2 & After harvest \\
\hline \multirow[t]{2}{*}{11} & Thousand kernel weight & Tkw_S1, Tkw_S2 & TkwD_S1, TkwD_S2 & RTkw_S1, RTkw_S2 & After harvest \\
\hline & Traits & Control & Drought & Time & \\
\hline \multicolumn{6}{|c|}{ Yield and Related Traits } \\
\hline 12 & Biomass & Bm_S1, Bm_S2 & BmD_S1, BmD_S2 & After harvest & \\
\hline 13 & Yield & Yd_S1,Yd_S2 & YdD_S1,YdD_S2 & After harvest & \\
\hline \multirow[t]{2}{*}{14} & Harvest Index & HI_S1,HI_S2 & HID_S1, HID_S2 & After harvest & \\
\hline & Index & Abbrev. & Formula & Time & \\
\hline \multicolumn{6}{|c|}{ Stress Indices } \\
\hline 15 & $\begin{array}{l}\text { Stress Tolerance Index } \\
\text { (STI) }\end{array}$ & STI_S1,STI_S2 & $\frac{(Y p \times Y s)}{(\overline{Y p})^{2}}$ & After harvest & \\
\hline 16 & Mean Productivity (MP) & MP_S1, MP_S2 & $\frac{\left(Y_{p}+Y_{s}\right)}{2}$ & After harvest & \\
\hline 17 & Stress Tolerance (ST) & ST_S1, ST_S2 & $Y p-Y_{s}$ & After harvest & \\
\hline 18 & $\begin{array}{l}\text { Stress Susceptibility Index } \\
\text { (SSI) }\end{array}$ & SSI_S1,SSI_S2 & {$\left[1-\left(\frac{Y_{s}}{Y_{p}}\right)\right] /\left[1-\left(\frac{\overline{Y_{s}}}{\overline{Y_{p}}}\right)\right]$} & After harvest & \\
\hline 19 & $\begin{array}{l}\text { Drought Resistance Index } \\
\text { (DRI) }\end{array}$ & DRI_S1, DR_S2 & $Y_{S} \times\left(\frac{Y_{S}}{Y_{p}}\right) / \overline{Y_{S}}$ & After harvest & \\
\hline
\end{tabular}

where $Y_{s}=$ yield under stress, $\overline{Y_{S}}=$ mean yield under stress, $Y p=$ yield under control and $\overline{Y_{p}}=$ mean yield under control

\section{Conclusions}

In the present study, we genetically dissected the performance of durum wheat RIL population for yield and yield-related traits and various morpho-physiological characteristics under irrigated and drought conditions through QTL mapping, which was mapped with the 265 assays. The experiments were conducted in two consecutive seasons at the exact same location to avoid variation due to soil properties and ground factor. A total of 221 QTLs were identified, where 145 QTLs were co-located with each other, and where 20 markers were associated with any trait in either S1 or S2. Furthermore, certain Yd QTLs match exactly with Gr, Tr, Hd, Tkw, HI, Sps and stress index QTLs, a finding consistent with previous reports. Several QTLs can be targeted for yield enhancement in durum wheat; the most promising ones from this study could be the clusters 2A.4 and 7B.5, where markers Xgwm895 and $X$ barc276, if combined, might increase the yield up to $11 \%$. In addition, an indirect improvement in yield is also possible by improving other traits ( $\mathrm{Tr}$, Tkw and HI), as we have found co-located QTLs for the said traits with Yd. Meanwhile, more studies are needed to validate our findings, our study still provides new insights into the genetic architecture of yield and related traits in durum wheat and the RILs with favorable loci could be used to improve yield in durum wheat.

Supplementary Materials: Supplementary materials can be found at http://www.mdpi.com/1422-0067/21/7/2372/s1.

Author Contributions: Conceptualization, M.A.R.A., S.S. and A.B.; Formal analysis, M.A.R.A.; Funding acquisition, M.A.R.A. and A.B.; Investigation, F.A., S.S., S.A. and M.Q.W.; Methodology, F.A., S.S., S.A., M.Q.W. and A.A.; Supervision, A.A.; Writing-original draft, M.A.R.A. and A.A.; Writing-review \& editing, A.B. All authors have read and agreed to the published version of the manuscript.

Funding: This research was funded by International Foundation for Science (IFS, Sweden), grant number C-5897-I.

Acknowledgments: The authors would also like to thank Sultan Mehmood Khan and Manzoor Ahmad for excellent technical assistance.

Conflicts of Interest: The authors declare no conflict of interest. The funders had no role in the design of the study; in the collection, analyses, or interpretation of data; in the writing of the manuscript, or in the decision to publish the results. 


\section{Abbreviations}

$\begin{array}{ll}\text { QTL } & \text { Quantitative trait loci } \\ \text { LOD } & \text { Log of odds } \\ \text { S1 } & \text { Season 1 (2016-17) } \\ \text { S2 } & \text { Season 2 (2017-18) } \\ \text { Gr } & \text { Germination } \\ \text { Hd } & \text { Heading time } \\ \text { Ph } & \text { Plant height } \\ \text { Tr } & \text { Transpiration rate } \\ \text { Phr } & \text { Photosynthetic rate } \\ \text { Sc } & \text { Stomatal conductance } \\ \text { Wue } & \text { Water use efficiency } \\ \text { Sl } & \text { Spike length } \\ \text { Sw } & \text { Spike weight } \\ \text { Sps } & \text { Seeds per spike } \\ \text { Tkw } & \text { Thousand kernel weight } \\ \text { Bm } & \text { Biomass } \\ \text { Yd } & \text { Yield } \\ \text { HI } & \text { Harvest index } \\ \text { STI } & \text { Stress tolerance index } \\ \text { SSI } & \text { Stress susceptibility index } \\ \text { MP } & \text { Mean productivity } \\ \text { DRI } & \text { Drought resistance index } \\ \text { ST } & \text { Stress tolerance }\end{array}$

\section{References}

1. Lev-Yadun, S.; Gopher, A.; Abbo, S. The cradle of agriculture. Science 2000, 288, 1602-1603. [CrossRef] [PubMed]

2. Tilman, D.; Balzer, C.; Hill, J.; Befort, B.L. Global food demand and the sustainable intensification of agriculture. Proc. Nat. Acad. Sci. USA 2011, 108, 20260-20264. [CrossRef] [PubMed]

3. Breakout session P1.1 National Food Security-The Wheat Initiative-an International Research Initiative for Wheat Improvement. Available online: http://www.fao.org/docs/eims/upload/306175/Briefing\%20Paper\% 20(3)-Wheat\%20Initative\%20-\%20H\%C3\%A91\%C3\%A8ne\%20Lucas.pdf (accessed on 22 December 2019).

4. Ames, N.P.; Clarke, J.M.; Marchylo, B.A.; Dexter, J.E.; Woods, S.M. Effect of environment and genotype on durum wheat gluten strength and pasta viscoelasticity. Cereal Chem. 1999, 76, 582-586. [CrossRef]

5. World Pasta Market and Turkey. Available online: http://www.magazinebbm.com/english/world-pastamarket-and-turkey/.html (accessed on 22 December 2019).

6. International Grains Council. Available online: http://www.igc.int/en/Default.aspx (accessed on 22 December 2019).

7. Araus, J.L.; Bort, J.; Steduto, P.; Villegas, D.; Royo, C. Breeding cereals for Mediterranean conditions: ecophysiological clues for biotechnology application. Ann. Appl. Biol. 2003, 142, 129-141. [CrossRef]

8. Araus, J.L.; Slafer, G.A.; Reynolds, M.P.; Royo, C. Plant breeding and drought in C3 cereals: what should we breed for? Ann. Bot. 2002, 89, 925-940. [CrossRef] [PubMed]

9. Condon, A.G.; Richards, R.A.; Rebetzke, G.J.; Farquhar, G.D. Breeding for high water-use efficiency. J. Exp. Bot. 2004, 55, 2447-2460. [CrossRef]

10. Ludlow, M.M.; Muchow, R.C. A critical evaluation of traits for improving crop yields in water-limited environments. Adv. Agron. 1990, 43, 107-153.

11. Rajaram, S. Prospects and promise of wheat breeding in the 21st century. In Wheat in a Global Environment; Springer: Dordrecht, The Netherlands, 2001; pp. 37-52.

12. Ashraf, M.; Harris, P. Abiotic Stresses: Plant Resistance through Breeding and Molecular Approaches; CRC Press: Boca Raton, FL, USA, 2005.

13. Sangtarash, M.H. Responses of different wheat genotypes to drought stress applied at different growth stages. Pak. J. Biol. Sci. 2010, 13, 114-119. [CrossRef] 
14. Clarke, J.M.; Richards, R.A.; Condon, A.G. Effect of drought stress on residual transpiration and its relationship with water use of wheat. Can. J. Plant Sci. 1991, 71, 695-702. [CrossRef]

15. Blum, A. Plant Breeding for Stress Environments; CRC Press: Boca Raton, FL, USA, 1998.

16. Zaharieva, M.; Gaulin, E.; Havaux, M.; Acevedo, E.; Monneveux, P. Drought and heat responses in the wild wheat relative Aegilops geniculata Roth. Crop Sci. 2001, 41, 1321-1329. [CrossRef]

17. Nachit, M.M.; Elouafi, I. Durum wheat adaptation in the Mediterranean dryland: Breeding, stress physiology, and molecular markers. In Challenges and Strategies of Dryland Agriculture, (Challengesandst); Crop Science Society of America and American Society of Agronomy: Madison, WI, USA, 2004; pp. 203-218, CSSA special Publication no. 32.

18. Houshmand, S.; Arzani, A.; Mirmohammadi-Maibody, S.A.M. Effects of salinity and drought stress on grain quality of durum wheat. Commun. Soil Sci. Plant 2014, 45, 297-308. [CrossRef]

19. Royo, C.; Di Fonzo, N.; Nachit, M.M.; Araus, J.L. Enhancing genetic grain yield potential and yield stability in durum wheat. In Proceedings of the Seminar Durum Wheat Improvement in the Mediterranean Region: New Challenges. Options Méditerranéennes, Zaragoza, Spain, 12-14 April 2000; Volume 40, pp. 88-93.

20. Mitchell, J.; Siamhan, D.M.; Wamala, M.; Risimeri, J.E.; Chinyamakobvu, E.; Henderson, S.; Fukai, S. The use of seedling leaf death score for evaluation of drought resistance of rice. Field Crop Res. 1998, 55, 129-139. [CrossRef]

21. Akram, M. Growth and yield components of wheat under water stress of different growth stages. Ban. J. Agric. Res. 2011, 36, 455-468. [CrossRef]

22. Khakwani, A.A.; Dennett, M.; Munir, M. Drought tolerance screening of wheat varieties by inducing water stress conditions. Songklanakarin J. Sci. Technol. 2011, 33, 135-142.

23. Ramirez-Vallejo, P.; Kelly, J.D. Traits related to drought resistance in common bean. Euphytica 1998, 99, 127-136. [CrossRef]

24. Mitra, J. Genetics and genetic improvement of drought resistance in crop plants. Curr. Sci. 2001, 80, 758-763.

25. Fernandez, G.C. Effective selection criteria for assessing plant stress tolerance. In Proceedings of the International Symposium on Adaptation of Vegetables and other Food Crops in Temperature and Water Stress, Shanhua, Taiwan, 13-16 August 1992; pp. 257-270.

26. Hall, A. Is dehydration tolerance relevant to genotypic difference in leaf senescence and crop adaption to dry environments. In Plant Responses to Cellular Dehydration during Environmental Stress; Close, T.J., Bray, E.A., Eds.; The American Soc. Plant Pathologists: Rockville, MD, USA, 1993; pp. 1-10.

27. Blanco, A.; Bellomo, M.; Cenci, A.; De Giovanni, C.; D’ovidio, R.; Iacono, E.; Laddomada, B.; Pagnotta, M.; Porceddu, E.; Sciancalepore, A. A genetic linkage map of durum wheat. Theor. Appl. Genet. 1998, 97, 721-728. [CrossRef]

28. Nachit, M.M.; Elouafi, I.; Pagnotta, M.A.; El-Saleh, A.; Iacono, E.; Labhilili, M.; Asbati, A.; Azrak, M.; Hazzam, H.; Benscher, D.; et al. Molecular linkage map for an intraspecific recombinant inbred population of durum wheat (Triticum turgidum L. var. durum). Theor. Appl. Genet. 2001, 102, 177-186. [CrossRef]

29. Maccaferri, M.; Sanguineti, M.C.; Corneti, S.; Ortega, J.L.A.; Salem, M.B.; Bort, J.; DeAmbrogio, E.; del Moral, L.F.G.; Demontis, A.; El-Ahmed, A.; et al. Quantitative trait loci for grain yield and adaptation of durum wheat (Triticum durum Desf.) across a wide range of water availability. Genetics 2008, 178, 489-511. [CrossRef]

30. Sourdille, P.; Perretant, M.; Charmet, G.; Leroy, P.; Gautier, M.; Joudrier, P.; Nelson, J.; Sorrells, M.E.; Bernard, M. Linkage between RFLP markers and genes affecting kernel hardness in wheat. Theor. Appl. Genet. 1996, 93, 580-586. [CrossRef]

31. Nauraein, M.; Mohammadi, S.A.; Ahrizad, S.; Moghaddam, M.; Sadeghzadeh, B. Evaluation of drought tolerance indices in wheat recombinant inbred population. Ann. Biol. Res. 2013, 4, 113-122.

32. Börner, A.; Schumann, E.; Fürste, A.; Cöster, H.; Leithold, B.; Röder, M.S.; Weber, W.E. Mapping of quantitative trait loci determining agronomic important characters in hexaploid wheat (Triticum aestivum L.). Theor. Appl. Genet. 2002, 105, 921-936. [CrossRef] [PubMed]

33. Dolferus, R.; Ji, X.M.; Richards, R.A. Abiotic stress control of grain number in cereals. Plant Sci. 2011, 181, 331-347. [CrossRef] [PubMed]

34. Bennett, D.; Reynolds, M.; Mullan, D.; Izanloo, A.; Kuchel, H.; Langridge, P.; Schnurbusch, T. Detection of two major grain yield QTL in bread wheat (Triticum aestivum L.) under heat, drought and high yield potential environments. Theor. Appl. Genet. 2012, 173, 1473-1485. [CrossRef] [PubMed]

35. Rehman-Arif, M.A.; Börner, A. Mapping of QTL associated with seed longevity in durum wheat (Triticum durum Desf.). J. Appl. Genet. 2019, 60, 33-36. [CrossRef] [PubMed] 
36. Peleg, Z.V.I.; Fahima, T.; Krugman, T.; Abbo, S.; Yakir, D.A.N.; Korol, A.B.; Saranga, Y. Genomic dissection of drought resistance in durum wheat $\times$ wild emmer wheat recombinant inbreed line population. Plant Cell Environ. 2009, 32, 758-779. [CrossRef] [PubMed]

37. Panio, G.; Motzo, R.; Mastrangelo, A.M.; Marone, D.; Cattivelli, L.; Giunta, F.; De Vita, P. Molecular mapping of stomatal-conductance-related traits in durum wheat (Triticum turgidum ssp. durum). Ann. Appl. Biol. 2013, 162, 258-270. [CrossRef]

38. Xu, Y.F.; Li, S.S.; Li, L.H.; Ma, F.F.; Fu, X.Y.; Shi, Z.L.; Xu, H.X.; Ma, P.T.; An, D.G. QTL mapping for yield and photosynthetic related traits under different water regimes in wheat. Mol. Breed. 2017, 37, 34. [CrossRef]

39. Hu, J.; Wang, X.; Zhang, G.; Jiang, P.; Chen, W.; Hao, Y.; Ma, X.; Xu, S.; Jia, J.; Kong, L.; et al. QTL mapping for yield-related traits in wheat based on four RIL populations. Theor. Appl. Genet. 2020, 133, 917-933. [CrossRef]

40. Nagel, M.; Navakode, S.; Scheibal, V.; Baum, M.; Nachit, M.; Röder, M.S.; Börner, A. The genetic basis of durum wheat germination and seedling growth under osmotic stress. Biol. Plant. 2014, 58, 681-688. [CrossRef]

41. Wang, S.G.; Jia, S.S.; Sun, D.Z.; Wang, H.Y.; Dong, F.F.; Ma, H.X.; Jing, R.L.; Ma, G. Genetic basis of traits related to stomatal conductance in wheat cultivars in response to drought stress. Photosynthetica 2015, 53, 299-305. [CrossRef]

42. Blanco, A.; Simeone, R.; Gadaleta, A. Detection of QTL for grain protein content in durum wheat. Theor. Appl. Genet. 2006, 112, 1195-1204. [CrossRef] [PubMed]

43. Patil, R.M.; Tamhankar, S.A.; Oak, M.D.; Raut, A.L.; Honrao, B.K.; Rao, V.S.; Misra, S.C. Mapping of QTL for agronomic traits and kernel characters in durum wheat (Triticum durum Desf.). Euphytica 2013, 190, 117-129. [CrossRef]

44. Kumar, N.; Kulwal, P.L.; Balyan, H.S.; Gupta, P.K. QTL mapping for yield and yield contibuting traits in two mapping populations fo bread wheat. Mol. Breed. 2007, 19, 163-177. [CrossRef]

45. Blanco, A.; Mangini, G.; Giancaspro, A.; Giove, S.; Colasuonno, P.; Simeone, R.; Signorile, A.; De Vita, P.; Mastrangelo, A.M.; Cattivelli, L.; et al. Relationships between grain protein content and grain yield components through quantitative trait locus analyses in a recombinant inbred line population derived from two elite durum wheat cultivars. Mol. Breed. 2012, 30, 79-92. [CrossRef]

46. Roncallo, P.F.; Akkiraju, P.C.; Cervigni, G.L.; Echenique, V.C. QTL mapping and analysis of epistatic interactions for grain yield and yield-related traits in Triticum turgidum L. var. durum. Euphytica 2017, 213, 277. [CrossRef]

47. Cuthbert, J.L.; Somers, D.J.; Brûlé-Babel, A.L.; Brown, P.D.; Crow, G.H. Molecular mapping of quantitative trait loci for yield and yield components in spring wheat (Triticum aestivum L.). Theo. Appl. Genet. 2008, 117, 595-608. [CrossRef]

48. Ain, Q.; Rasheed, A.; Anwar, A.; Mahmood, T.; Imtiaz, M.; Xia, X.; He, Z.; Quraishi, U.M. Genome-wide association for grain yield under rainfed conditions in historical wheat cultivars from Pakistan. Front. Plant Sci. 2015, 6, 743. [CrossRef]

49. Qaseem, M.F.; Qureshi, R.; Shaheen, H.; Shafqat, N. Genome-wide association analyses for yield and yield-related traits in bread wheat (Triticum aestivum L.) under pre-anthesis combined heat and drought stress in field conditions. PloS ONE 2019, 14, e213407. [CrossRef]

50. Golabadi, M.A.; Arzani, A.; Mirmohammadi-Maibody, S.A.M. Assessment of drought tolerance in segregating populations in durum wheat. Afr. J. Agric. Res. 2006, 5, 162-171.

51. Ballesta, P.; Freddy Mora, F.; Del-Pozo, A. Association mapping of drought tolerance indices in wheat: QTL-rich regions on chromosome 4A. Sci. Agricola 2020, 77, 2. [CrossRef]

52. Golabadi, M.; Arzani, A.; Mirmohammadi-Maibody, S.A.M.; Sayed-Tabatabaei, B.E.; Mohammadi, S.A. Identification of microsatellite markers linked with yield components under drought stress at terminal growth stages in durum wheat. Euphytica 2011, 177, 207-221. [CrossRef]

53. Wang, S.; Basten, C.J.; Zeng, Z.B. Windows QTL Cartographer 2.5. Department of Statistics, North Carolina State University, Raleigh. Available online: http://statgen.ncsu.edu/qtlcart/WQTLCart.html (accessed on 22 December 2019).

(C) 2020 by the authors. Licensee MDPI, Basel, Switzerland. This article is an open access article distributed under the terms and conditions of the Creative Commons Attribution (CC BY) license (http://creativecommons.org/licenses/by/4.0/). 